\title{
A Flexible Search Framework for CHR
}

\author{
Leslie De Koninck ${ }^{\star}$, Tom Schrijvers ${ }^{\star \star}$, and Bart Demoen \\ Department of Computer Science, K.U.Leuven, Belgium \\ FirstName. LastName @cs. kuleuven. be
}

\begin{abstract}
This paper introduces a framework for the specification of tree search strategies in $\mathrm{CHR}$ with disjunction $\left(\mathrm{CHR}^{\vee}\right)$. We support the specification of common search strategies such as depth-first, breadthfirst and best-first, as well as constrained optimization by means of branch \& bound search. The framework is given as an extension of CHR with rule priorities $\left(\mathrm{CHR}^{\mathrm{rp}}\right)$ in which each branch of the search tree is assigned a branch priority. This approach leads to a uniform solution to execution control in CHR.
\end{abstract}

\section{Introduction}

Constraint Handling Rules (CHR) [12] is a high-level rule-based language, designed for the implementation of constraint solvers. It runs on top of a host language like Prolog [26], Java [4,33], Haskell or Curry [16], which provides a built-in constraint solver supporting at least a syntactic equality constraint, as well as the constraints true and false.

CHR aims at being a high-level language for implementing constraint solvers. Indeed, it is excellent at representing the propagation logic of constraint solvers: the notion of a constraint propagator corresponds with a CHR rule. The CHR language extension [5] also presents a high-level means to express the search aspect of constraint solving. However, due to the non-deterministic operational semantics of these features, CHR is but an abstraction of constraint solving. Most constraint problems are of too big a size to be naively entrusted to a nondeterministic solving process. Rather, solving strategies must be specified to cleverly direct the solving process and prune the search space early and eagerly. An appropriate solving strategy can indeed reduce the solving cost by many orders of magnitude and make the difference between an infeasible and practical approach.

It is for this reason that most state-of-the-art constraint solvers offer the means to select and/or specify the desired solving strategy. For instance, there are 18 documented options, many of which are parameterized and/or can be combined, to influence the strategy of the enumeration predicate labeling/2

\footnotetext{
* Research funded by a Ph.D. grant of the Institute for the Promotion of Innovation through Science and Technology in Flanders (IWT-Vlaanderen)

** Post-Doctoral Researcher of the Fund for Scientific Research - Flanders (Belgium) (F.W.O. - Vlaanderen)
} 
in the clp(fd) library of SICStus Prolog [7]. The solving strategy often falls apart in two distinct aspects: propagator priorities for conjunctions and search priorities for disjunctions. Propagator priorities can be specified by means of rule priorities: these have been studied in $\mathrm{CHR}^{\mathrm{rp}}$ [10]. In this paper, we add the missing piece: search priorities.

The main contributions of this paper are the following:

1. We present $\mathrm{CHR}_{\vee}^{\text {brp }}$, a high-level approach for specifying the control flow in $\mathrm{CHR}^{\vee}$ (Section 3). $\mathrm{CHR}_{\vee}^{\mathrm{brp}}$ extends $\mathrm{CHR}^{\vee}$ with both $\mathbf{b}$ ranch and $\mathbf{r}$ ule priorities.

2. We show how to express standard tree search strategies such as depth-first, breadth-first, depth-first iterative deepening and limited discrepancy search in $\mathrm{CHR}_{\vee}^{\mathrm{brp}}$ (Section 4).

3. We show how conflict-directed backjumping can be realized by extending our framework with justifications (Section 5). Our work extends [38] by not restricting the search strategy to left-to-right depth-first, and by addressing correctness and optimality.

This work is based on previous work in [9]. There are three main improvements. Firstly, whereas in [9], the search strategy is determined by choosing an appropriate definition of the Split transition, in this work, the search strategy is determined by the program. This allows using program dependent information for informed search, for example for a best-first search strategy. Secondly, our search framework is based on $\mathrm{CHR}^{\mathrm{rp}}$ and the $\omega_{p}$ semantics, while in [9], it is based on the more low-level $\omega_{r}$ semantics of CHR. Finally, we also consider constrained optimization, a topic that hitherto has not been tackled.

\section{CHR with Rule Priorities and Disjunction}

In this section, we introduce both $\mathrm{CHR}^{\mathrm{rp}}$, CHR with rule priorities [10] (Section 2.2), and $\mathrm{CHR}^{\vee}$, CHR with disjunctive rule bodies [5] (Section 2.3). We first review the syntax and semantics of regular CHR in Section 2.1.

\subsection{Constraint Handling Rules}

Syntax A constraint $c\left(t_{1}, \ldots, t_{n}\right)$ is an atom of predicate $c / n$ with $t_{i}$ a host language value (e.g., a Herbrand term in Prolog) for $1 \leq i \leq n$. There are two types of constraints: built-in constraints and CHR constraints (also called user-defined constraints). The CHR constraints are solved by the CHR program whereas the built-in constraints are solved by an underlying constraint solver (e.g., the Prolog unification algorithm).

There are three types of Constraint Handling Rules: simplification rules, propagation rules and simpagation rules. They have the following form:

$$
\begin{array}{lll}
\text { Simplification } r @ & H^{r} \Longleftrightarrow g \mid B \\
\text { Propagation } r @ H^{k} & \Longleftrightarrow g \mid B \\
\text { Simpagation } r @ H^{k} \backslash H^{r} \Longleftrightarrow g \mid B
\end{array}
$$


where $r$ is the rule name, $H^{k}$ and $H^{r}$ are non-empty sequences of CHR constraints and are called the heads of the rule, the rule guard $g$ is a conjunction of built-in constraints, and the rule body $B$ is a multi-set of both CHR and built-in constraints. Throughout this text, in particular in the descriptions of the operational semantics, we use the simpagation rule form to denote any type of rule, where $H^{k}$ is empty in case of a simplification rule, and $H^{r}$ is empty in case of a propagation rule. A program $P$ is a set of $\mathrm{CHR}$ rules.

The theoretical operational semantics Operationally, CHR constraints have multi-set semantics. To distinguish between different occurrences of syntactically equal constraints, CHR constraints are extended with a unique identifier. An identified CHR constraint is denoted by $c \# i$ with $c$ a CHR constraint and $i$ the identifier. We write $\operatorname{chr}(c \# i)=c$ and $\operatorname{id}(c \# i)=i$. The theoretical operational semantics of CHR, denoted $\omega_{t}$, is given in [11] as a state transition system. A CHR execution state $\sigma$ is represented as a tuple $\langle G, S, B, T\rangle_{n}$ where $G$ is the goal, a multi-set of constraints that need to be solved; $S$ is the CHR constraint store, a set of identified CHR constraints; $B$ is the built-in constraint store, a conjunction of built-in constraints; $T$ is the propagation history, a set of tuples denoting the rule instances that have already fired; and $n$ is the next free identifier, used to identify new CHR constraints. The transitions of $\omega_{t}$ are shown in Table 1 , where $\mathcal{D}$ denotes the built-in constraint theory, $\bar{\exists}_{X} Y$ denotes the existential closure of $Y$ apart from the variables appearing in $X$, and $\uplus$ is the multi-set union operation which we also use for sets in case a disjoint union is required.

1. Solve $\langle\{c\} \uplus G, S, B, T\rangle_{n} \stackrel{\omega_{t}}{\longmapsto_{P}}\langle G, S, c \wedge B, T\rangle_{n}$ where $c$ is a built-in constraint.

2. Introduce $\langle\{c\} \uplus G, S, B, T\rangle_{n} \stackrel{\omega_{t}}{\longmapsto} P\langle G,\{c \# n\} \cup S, B, T\rangle_{n+1}$ where $c$ is a CHR constraint.

3. Apply $\left\langle G, H_{1} \uplus H_{2} \uplus S, B, T\right\rangle_{n} \stackrel{\omega_{t}}{\longmapsto_{P}}\left\langle C \uplus G, H_{1} \cup S, \theta \wedge B, T \cup\{t\}\right\rangle_{n}$ where $P$ contains a (renamed apart) rule

$$
r @ H_{1}^{\prime} \backslash H_{2}^{\prime} \Longleftrightarrow g \mid C
$$

and a matching substitution $\theta$ such that $\operatorname{chr}\left(H_{1}\right)=\theta\left(H_{1}^{\prime}\right), \operatorname{chr}\left(H_{2}\right)=\theta\left(H_{2}^{\prime}\right)$, $\mathcal{D} \models B \rightarrow \bar{\exists}_{B}(\theta \wedge g)$, and $t=\left\langle\operatorname{id}\left(H_{1}\right), \operatorname{id}\left(H_{2}\right), r\right\rangle \notin T$.

Table 1. Transitions of $\omega_{t}$

The Solve transition solves a built-in constraint from the goal, the Introduce transition inserts a new CHR constraint from the goal into the CHR constraint store, and the Apply transition fires a rule instance. A rule instance $\theta(r)$ instantiates a rule with CHR constraints matching the heads, using matching substitution $\theta$. A state is called final if no transition applies to it. 
The refined operational semantics The refined operational semantics of CHR, denoted by $\omega_{r}$, is introduced in [11] as a formalization of the execution mechanism of most current CHR implementations. The $\omega_{r}$ semantics is based on the concept of an active constraint. The active constraint is a CHR constraint that is used as a starting point for finding applicable rule instances. To ensure that all rule instances are eventually tried, all new CHR constraints become active after they are asserted. CHR constraints that have been active before and whose variables are affected by a new built-in constraint, are reactivated.

The active constraint tries rules in textual order until either it finds a applicable rule instance or all rules have been tried. When a rule instance fires, its body is processed from left to right. Every new CHR constraint that is processed, is activated as soon as it is inserted into the constraint store. Every new built-in constraint is solved for, and all affected CHR constraints are activated one by one before processing the next constraint in the body. If the active constraint has not been removed, then after processing the rule body, it searches for the next applicable rule instance. Otherwise, processing resumes where it left before the constraint was activated.

Many programs rely on the execution order imposed by the $\omega_{r}$ semantics. In Section 2.2, we present an alternative to the $\omega_{r}$ semantics that offers a more high-level and flexible form of execution control.

\subsection{CHR with Rule Priorities}

CHR $^{\text {rp }}$ extends CHR with user-defined rule priorities. It is introduced in [10] to support more high-level and flexible execution control than previously available in CHR by means of the low-level $\omega_{r}$ semantics, while retaining the expressive power needed for the implementation of general purpose algorithms.

Syntax The syntax of $\mathrm{CHR}^{\mathrm{rp}}$ is compatible with the syntax of (regular) CHR. A CHR ${ }^{\mathrm{rp}}$ simpagation rule looks as follows:

$$
p:: r @ H^{k} \backslash H^{r} \Longleftrightarrow g \mid B
$$

where $r, H^{k}, H^{r}, g$ and $B$ are as defined in Section 2.1. The rule priority $p$ is an arithmetic expression for which holds that $\operatorname{vars}(p) \subseteq\left(\operatorname{vars}\left(H^{k}\right) \cup \operatorname{vars}\left(H^{r}\right)\right)$, i.e., all variables in $p$ also appear in the heads. A rule in which $\operatorname{vars}(p)=\emptyset$ is called a static priority rule: its priority is known at compile time and is the same for all its instances. A rule in which $\operatorname{vars}(p) \neq \emptyset$ is called a dynamic priority rule: its priority is only known at runtime and different instances of the same rule may fire at different priorities. We say that priority $p$ is higher than priority $p^{\prime}$ if $p<p^{\prime}$.

The priority semantics The operational semantics of $\mathrm{CHR}^{\mathrm{rp}}$ is called the priority semantics and is denoted by $\omega_{p}$. It consists of a refinement of the $\omega_{t}$ semantics with a minimal amount of determinism in order to support rule priorities. The $\omega_{p}$ semantics uses the same state representation as the $\omega_{t}$ semantics. 
It restricts the applicability of the Apply transition with respect to the $\omega_{t}$ semantics: this transition is only applicable to states with an empty goal and it fires a rule instance of priority $p$ in state $\sigma$ only if there exists no $\omega_{t}$ Apply transition $\sigma \stackrel{\omega_{t}}{\longmapsto} P \sigma^{\prime}$ that fires a rule instance with a higher priority. The Solve and Introduce transitions are unchanged w.r.t. the $\omega_{t}$ semantics.

\subsection{CHR with Disjunction}

Constraint Handling Rules is extended with disjunctions in rule bodies in [5] (see also [2] and [3, Chapter 5]). The resulting language is denoted by $\mathrm{CHR}^{\vee}$. The syntax of $\mathrm{CHR}^{\vee}$ is the same as that of regular CHR, except that rule bodies are formulas built from atoms by conjunctions and disjunctions. In [9], we define a theoretical operational semantics $\omega_{t}^{\vee}$ for $\mathrm{CHR}^{\vee}$, which extends the $\omega_{t}$ semantics of CHR. An $\omega_{t}^{\vee}$ execution state is a multi-set $\Sigma=\left\{\sigma_{1}, \ldots, \sigma_{n}\right\}$ of $\omega_{t}$ execution states. Each element $\sigma_{i} \in \Sigma$ represents an alternative solution. The following transitions are defined on $\omega_{t}^{\vee}$ executions states:

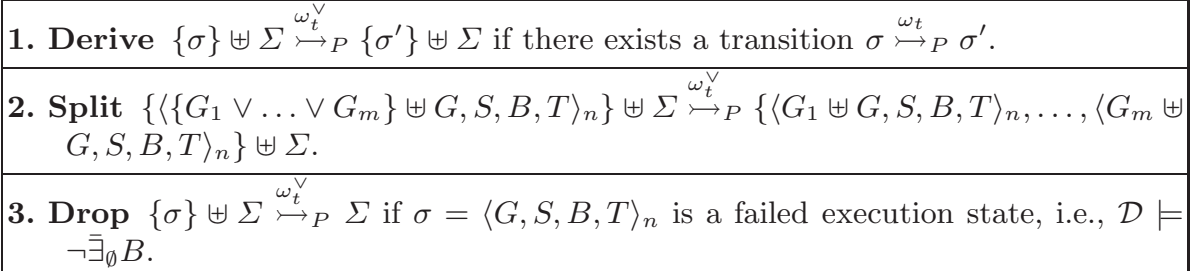

Table 2. Transitions of $\omega_{t}^{\vee}$

The Drop transition is new compared to our description in [9]. It removes failed alternatives from the search tree and is introduced to support pruning of the search tree, for example during conflict-directed backjumping (see Section 5). This pruning respects the declarative semantics of $\mathrm{CHR}^{\vee}$ (see [3, Chapter 5]).

Example 1 (4-queens). A solver for the 4-queens problem can be written in $\mathrm{CHR}^{\vee}$ as follows.

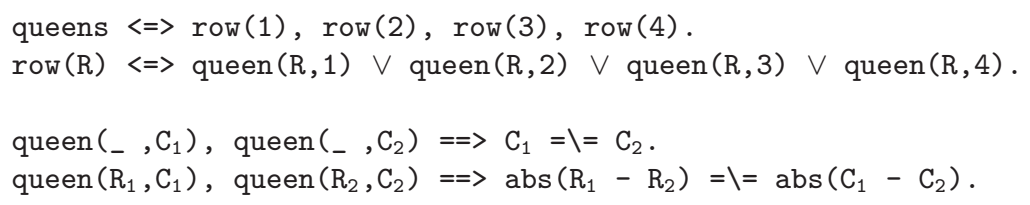

As goal we use \{queens\}. Here, a queen $(R, C)$ constraint means that there is a queen placed on the field with row $R$ and column $C$. The first rule states that there are four rows. The second rule states that there is a queen in one of the columns for each row. The remaining two rules ensure that no two queens 
are in conflicting positions. The first of them makes sure that there are no two queens in the same column; the second that there are no two queens on the same diagonal. The program above can easily be adapted to solve the general $n$-queens problem.

Search trees An $\omega_{t}^{\vee}$ derivation can be visualized as a search tree. Such a search tree consists of a set of nodes and a set of (directed) edges connecting these nodes. A node is either an internal node or a leaf node. An internal node represents a choice point and corresponds to a state in which a Split transition applies. The root node corresponds to the initial state $\langle G, \emptyset, \text { true, } \emptyset\rangle_{1}$ with $G$ the initial goal. It can be considered an internal node corresponding to a choice point with only one alternative. A leaf node represents a successful or failed final $\omega_{t}$ execution state. An edge goes from one node, its start node, to another node, its end node, and represents the derivation that transforms one of the alternatives of its start node into its end node, i.e., it consists of a series of execution states that are linked by Derive transitions. For example, the derivation

$$
\left\{\sigma_{0}\right\} \stackrel{\omega_{t}^{\vee}}{\longmapsto} P\left\{\sigma_{1}, \sigma_{2}\right\} \stackrel{\omega_{t}^{\vee}}{\longmapsto} P\left\{\sigma_{3}, \sigma_{2}\right\} \stackrel{\omega_{t}^{\vee}}{\longmapsto} P\left\{\sigma_{3}, \sigma_{4}\right\} \stackrel{\omega_{t}^{\vee}}{\longmapsto} P\left\{\sigma_{3}, \sigma_{5}, \sigma_{6}, \sigma_{7}\right\}
$$

corresponds to the following search tree:

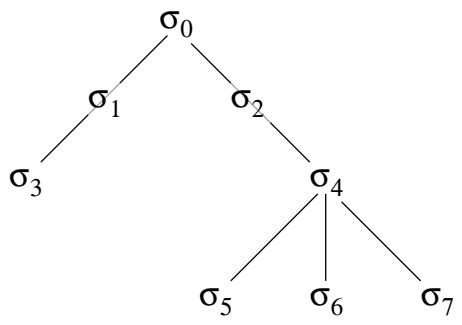

In this example case, the search tree is traversed in left-to-right, depth-first order. Note that different search trees are possible for the same goal. Consider for example the initial goal $\left\{\left(G_{1} \vee G_{2}\right),\left(G_{3} \vee G_{4}\right)\right\}$. For this goal, some derivations apply the Split transition to the subgoal $G_{1} \vee G_{2}$ first, while others apply this transition first to $G_{3} \vee G_{4}$.

\section{A Combined Approach}

In this section, we combine $\mathrm{CHR}^{\mathrm{rp}}$ with $\mathrm{CHR}^{\vee}$ into a flexible framework for defining both the search and propagation strategy to be used by the CHR constraint solver. While our previous work [9] extended the refined operational semantics of CHR with facilities for search strategy control, this work extends the more high-level priority semantics of $\mathrm{CHR}^{\mathrm{rp}}$.

First, we discuss some issues concerning search strategies and constrained optimization. A search strategy determines the order in which answers (solutions) to a problem are generated. This order is only relevant if we need a subset 
of all answers, in particular if we only need one. If we need all answers, then a simple sorting of these answers suffices to implement any search strategy. We assume here that search tree branches are processed independently; but see Section 5 for a discussion of conflict-directed backjumping, a search technique that does take into account results from other branches. In the case of naive optimization, implemented by computing all answers and then choosing the optimal one amongst these, the search strategy is of no importance. However, in a more intelligent form, using for example a branch $\&$ bound approach, a good search strategy may cause considerable pruning of the search tree.

In [9], the $\omega_{r}^{\vee}$ semantics states that only the first answer is derived. ${ }^{1}$ Other answers can be retrieved on external request (e.g., from the Prolog toplevel) by discarding the first answer and continuing search using the $\omega_{r}^{\vee}$ Next transition. Similarly, under the $\omega_{r}^{\vee^{*}}$ semantics presented in [38], the derivation stops as soon as the first answer is found, or the search tree is traversed completely. Retrieving the other answers is not explicitly supported, but such support can easily be added to the approach.

\subsection{An Intermediate Step: $\mathrm{CHR}_{\vee}^{\mathrm{rp}}$}

As an intermediate step, we introduce a simple combination of $\mathrm{CHR}^{\mathrm{rp}}$ with $\mathrm{CHR}^{\vee}$ into the combined language $\mathrm{CHR}_{\vee}^{\mathrm{rp}}$. This language supports execution control with respect to conjuncts by means of the $\mathrm{CHR}^{\mathrm{rp}}$ rule priorities, but leaves the search control undetermined. The syntax of $\mathrm{CHR}_{\vee}^{\mathrm{rp}}$ is similar to that of $\mathrm{CHR}^{\mathrm{rp}}$, but also allows disjunction in the rule bodies, like in $\mathrm{CHR}^{\vee}$. The operational semantics of $\mathrm{CHR}_{\vee}^{\mathrm{rp}}$ is almost the same as that of $\mathrm{CHR}^{\vee}$. The only difference is that in the Derive transition, $\omega_{t}$ is replaced by $\omega_{p}$. In the next subsection, we extend $\mathrm{CHR}_{\vee}^{\mathrm{rp}}$ with branch priorities to support the specification of search strategies. Finally, in Section 3.3, a correspondence result is given, relating $\mathrm{CHR}_{\vee}^{\text {brp }}$ programs and derivations to $\mathrm{CHR}^{\vee}$ programs and derivations.

\subsection{Extending $\mathrm{CHR}_{\vee}^{\mathrm{rp}}$ with Branch Priorities}

Syntax The syntax of $\mathrm{CHR}_{\vee}^{\mathrm{brp}}$ extends the syntax of $\mathrm{CHR}_{\vee}^{\mathrm{rp}}$ with branch priorities. A $\mathrm{CHR}_{\vee}^{\text {brp }}$ simpagation rule looks as follows:

$$
(b p, r p):: r @ H^{k} \backslash H^{r} \Longleftrightarrow g \mid b p_{1}:: B_{1} \vee \ldots \vee b p_{m}:: B_{m}
$$

where $r, H^{k}, H^{r}$ and $g$ are as defined in Section 2.1. The rule priority $r p$ is as in $\mathrm{CHR}^{\mathrm{rp}}$. The branch priority $b p$ is a term: there is no a priori reason to restrict the allowed terms. In the examples, we use (tuples of) (lists of) integers and variables.

The rule body consists of a set of disjuncts $B_{i}$, each of which is annotated with a branch priority $b p_{i}(1 \leq i \leq m)$. To simplify the presentation (e.g., of the correspondence result in Section 3.3), we impose that each disjunct $B_{i}$ is a

\footnotetext{
${ }^{1}$ An answer (or solution) is a successful final $\omega_{r}$ execution state.
} 
conjunction of constraints. In particular, we do not support nested disjunctions. If in a rule with a single disjunct, the branch priority of this disjunct equals the one of its parent branch, then the branch priorities can be omitted, and so each $\mathrm{CHR}^{\mathrm{rp}}$ rule is also a syntactically valid $\mathrm{CHR}_{\vee}^{\mathrm{brp}}$ rule.

A $\mathrm{CHR}_{\vee}^{\text {brp }}$ program is a tuple $\left\langle\mathcal{R}, \mathcal{B P}, b p_{0}, \preceq\right\rangle$ where $\mathcal{R}$ is a set of $\mathrm{CHR}_{\vee}^{\text {brp }}$ rules, $\mathcal{B P}$ is the domain of the branch priorities, $b p_{0} \in \mathcal{B P}$ is the initial branch priority, and $\preceq$ is a total preorder relation over elements of $\mathcal{B P} .{ }^{2}$

Operational semantics We extend the $\omega_{p}$ states with a branch priority. The combination is called an alternative and is denoted by $b p:: \sigma$ where $b p$ is the branch priority and $\sigma$ is an $\omega_{p}$ execution state. ${ }^{3}$ An alternative can be marked, in which case it is written as $b p:: \sigma^{\star}$. Marking is used to discard a solution in order to derive a next one.

The operational semantics $\omega_{p}^{\vee}$ considers (multi-)sets of alternatives. A total pre-order $\preceq$ must be defined on their branch priorities, so that an alternative with the highest priority can be determined. In practice, we most often use a total order and in the examples, we define $\preceq$ by a logical formula containing arithmetic expressions. This implies that certain parts of the branch priorities must be ground. For a set $\Sigma$ of alternatives, we denote by max_bp $(\Sigma)$ the highest branch priority of any unmarked alternative in $\Sigma$, or in case $\Sigma$ does not contain any unmarked alternatives, a branch priority that is smaller than any other branch priority. The transitions of $\omega_{p}^{\vee}$ are given in Table 3 .

The main differences w.r.t. the $\omega_{t}^{\vee}$ semantics are the following. The Derive transition is split up into three transitions corresponding to the $\omega_{p}$ transitions in order to support matching with, and guards involving the branch priority. These three transitions, as well as the Split and Drop transitions, only apply to the highest priority unmarked alternative. Finally, a new transition called Mark is introduced, whose purpose is to mark a solution (successful final state) in order to find a next solution. Given a goal $G$, we construct an initial $\omega_{p}^{\vee}$ state $\Sigma_{0}=\left\{b p_{0}::\langle G, \emptyset, \text { true }, \emptyset\rangle_{1}\right\}$ where $b p_{0}$ is the initial branch priority for the program.

Noteworthy is that the branch priorities presented above, do not change the shape of the search tree. Instead, they only influence the order in which the nodes of this search tree are explored. Therefore, the search strategy used is of importance only in the following cases.

- If we require a subset of all solutions.

- If we combine the search strategy with an intelligent backtracking technique such as conflict-directed backjumping (see Section 5).

- If we need an optimal solution using a branch \& bound or restart optimization approach.

\footnotetext{
${ }^{2}$ We do not require that $\preceq$ is antisymmetric, i.e., $x \preceq y \wedge y \preceq x$ does not imply $x=y$.

${ }^{3}$ In the following, we treat states in which the goal contains a disjunction as $\omega_{p}$ states, but we do not call them final states, even if no $\omega_{p}$ transition applies to them.
} 
1a. Solve $\left\{b p::\langle\{c\} \uplus G, S, B, T\rangle_{n}\right\} \uplus \Sigma \stackrel{\omega_{p}^{\vee}}{\longmapsto_{P}}\left\{b p \quad::\langle G, S, c \wedge B, T\rangle_{n}\right\} \uplus \Sigma$ if $\max \_\mathrm{bp}(\Sigma) \preceq b p$ and $c$ is a built-in constraint.

1b. Introduce $\left\{b p::\langle\{c\} \uplus G, S, B, T\rangle_{n}\right\} \uplus \Sigma \stackrel{\omega_{p}^{\vee}}{\longmapsto} P\left\{\left\{b::\langle G,\{c \# n\} \cup S, B, T\rangle_{n+1}\right\} \uplus\right.$ $\Sigma$ if $\max \_b p(\Sigma) \preceq b p$ and $c$ is a CHR constraint.

1c. Apply $\left\{b p::\left\langle\emptyset, H_{1} \uplus H_{2} \uplus S, B, T\right\rangle_{n}\right\} \uplus \Sigma \stackrel{\omega_{p}^{\vee}}{\longmapsto} P$ P $\left\{b p::\left\langle C, H_{1} \cup S, \theta \wedge B, T \cup\right.\right.$ $\left.\{t\}\rangle_{n}\right\} \uplus \Sigma$ if max_bp $(\Sigma) \preceq b p$ and where $P$ contains a rule of priority $r p$ of the form

$$
\left(b p^{\prime}, r p\right):: r @ H_{1}^{\prime} \backslash H_{2}^{\prime} \Longleftrightarrow g \mid C
$$

and a matching substitution $\theta$ exists such that $\operatorname{chr}\left(H_{1}\right)=\theta\left(H_{1}^{\prime}\right), \operatorname{chr}\left(H_{2}\right)=$ $\theta\left(H_{2}^{\prime}\right), b p=\theta\left(b p^{\prime}\right), \mathcal{D} \models B \rightarrow \bar{\exists}_{B}(\theta \wedge g), \theta(r p)$ is a ground arithmetic expression and $t=\left\langle\operatorname{id}\left(H_{1}\right), \operatorname{id}\left(H_{2}\right), r\right\rangle \notin T$. Furthermore, no rule of priority $r p^{\prime}$ and substitution $\theta^{\prime}$ exists with $\theta^{\prime}\left(r p^{\prime}\right)<\theta(r p)$ for which the above conditions hold.

2. Split $\{b p:: \sigma\} \uplus \Sigma \stackrel{\omega_{p}^{\vee}}{\longmapsto} P\left\{b p_{1}:: \sigma_{1}, \ldots, b p_{m}:: \sigma_{m}\right\} \uplus \Sigma$ if max_bp $(\Sigma) \preceq b p$ and where $\sigma=\left\langle\left\{b p_{1}:: G_{1} \vee \ldots \vee b p_{m}:: G_{m}\right\} \uplus G, S, B, T\right\rangle_{n}$ and $\sigma_{i}=\left\langle G_{i} \uplus G, S, B, T\right\rangle_{n}$ for $1 \leq i \leq m$.

3. Drop $\left\{b p::\langle G, S, B, T\rangle_{n}\right\} \uplus \Sigma \stackrel{\omega_{t}^{\vee}}{\mapsto_{P}} \Sigma$ if $\max \_b p(\Sigma) \preceq b p$ and $\mathcal{D} \models \neg \bar{\exists}_{\emptyset} B$.

4. Mark $\{b p:: \sigma\} \uplus \Sigma \stackrel{\omega_{p}^{\vee}}{\longmapsto} P\left\{b p:: \sigma^{\star}\right\} \uplus \Sigma$ if $\max \_b p(\Sigma) \preceq b p$ and no other $\omega_{p}^{\vee}$ transition applies.

Table 3. Transitions of $\omega_{p}^{\vee}$ 
In contrast, if we require all solutions and do not apply any pruning based on previously computed answers, then the search strategy is irrelevant. ${ }^{4}$

In general, we might be interested in retrieving solutions one at a time. This is for example supported in the Prolog context by means of the toplevel environment asking whether more solutions are needed. We define a function find_next that returns, given an $\omega_{p}^{\vee}$ state, the first answer (solution) in this state, as well as the resulting state after marking this solution, which contains the remaining answers.

$$
\text { find_next }(\Sigma)=\left\langle A, \Sigma_{R}\right\rangle
$$

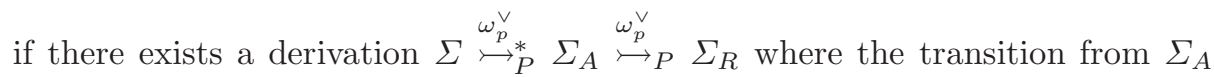
to $\Sigma_{R}$ is a Mark transition and the derivation from $\Sigma$ to $\Sigma_{A}$ does not contain such a transition. In the result, $A=B \wedge \operatorname{chr}(S)$ where $b p::\langle\emptyset, S, B, T\rangle_{n}$ is the highest priority unmarked alternative in $\Sigma_{A}$.

Constrained optimization As a general approach to constrained optimization, we show how both a branch \& bound, and restart optimization scheme can be implemented in $\mathrm{CHR}_{\vee}^{\text {brp }}$. We consider a goal $G$ whose best solution is to be returned. This best solution is such that there exists no solution that assigns a lower value to a given cost function $F$ whose variables appear in $G .{ }^{5}$ Let there be given an initial $\omega_{p}^{\vee}$ state $\Sigma_{0}$ based on the goal $G$. Under the assumption that there is a solution, we find the solution that minimizes our cost function $F$ as find_min $\left(\Sigma_{0}, F\right)$ where find_min is defined as

$$
\begin{aligned}
& \text { find_min }(\Sigma, F)= \text { let }\left\{\begin{array}{l}
\left\langle A, \Sigma^{\prime}\right\rangle=\text { find_next }(\Sigma) \\
\Sigma^{\prime \prime}=\text { add_goal }\left(F<F(A), \Sigma^{\prime}\right)
\end{array}\right. \\
& \text { in } \begin{aligned}
\text { if } \Sigma^{\prime \prime} \text { has no solution then } A \\
\text { else find_min }\left(\Sigma^{\prime \prime}, F\right)
\end{aligned}
\end{aligned}
$$

for branch \& bound optimization, and for restart optimization as follows:

$$
\begin{aligned}
& \text { find_min }(\Sigma, F)= \text { let }\left\{\begin{array}{l}
\left\langle A, \Sigma^{\prime}\right\rangle=\text { find_next }(\Sigma) \\
\Sigma^{\prime \prime}=\text { add_goal }(F<F(A), \Sigma)
\end{array}\right. \\
& \text { in } \begin{aligned}
\text { if } \Sigma^{\prime \prime} \text { has no solution then } A \\
\text { else find_min }\left(\Sigma^{\prime \prime}, F\right)
\end{aligned}
\end{aligned}
$$

Here add_goal $(G, \Sigma)$ returns the $\omega_{p}^{\vee}$ state that results from adding the goal $G$ to all unmarked alternatives in $\Sigma$ :

$$
\begin{aligned}
& \text { add_goal }(G, \Sigma)=\left\{b p:: \sigma^{\star} \mid b p:: \sigma^{\star} \in \Sigma\right\} \uplus \\
& \left\{b p::\left\langle G \uplus G^{\prime}, S, B, T\right\rangle_{n} \mid b p::\left\langle G^{\prime}, S, B, T\right\rangle_{n} \in \Sigma\right\}
\end{aligned}
$$

\footnotetext{
${ }^{4}$ This is related to the distinction between variable ordering and value ordering in case all solutions are required, see for example [30].

${ }^{5}$ Alternative, one can use a utility function that is to be maximized.
} 
The difference between both approaches is that in branch \& bound optimization, the new bound is added as a goal to all remaining alternatives, whereas in restart optimization, the new bound is added to the initial state, which is solved for again.

Dynamic search tree generation It is not always convenient or even possible to state at compile time which alternatives are to be created. In general, we may want to generate alternatives dynamically, at runtime. Let us assume we can compute, by means of a built-in constraint, a list of all alternatives to be generated, as well as a list of priorities to be used for the respective alternatives. We can then dynamically generate the alternatives by asserting a generate_alternatives $/ 2$ constraint whose arguments are respectively the list of priorities and the list of alternatives. Let $T \in \mathcal{B P}$ be higher than all branch priorities used for the dynamically generated alternatives. We can then define the generate_alternatives/2 constraint by the following rules.

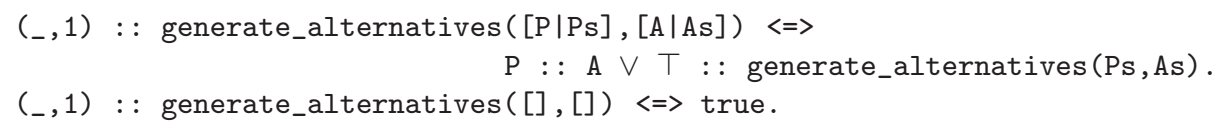

By using the $\top$ priority value, we ensure that all alternatives are generated before the first of them is processed.

\subsection{Correspondence}

In this subsection, we show that every $\omega_{p}^{\vee}$ derivation of a $\mathrm{CHR}_{\vee}^{\text {brp }} \operatorname{program} P$, corresponds to an $\omega_{t}^{\vee}$ derivation of a corresponding non-deterministic $\mathrm{CHR}^{\vee}$ version of this program $P$. We first show how to create such a non-deterministic version of a $\mathrm{CHR}_{\vee}^{\text {brp }}$ program. Next, we propose a mapping from $\omega_{p}^{\vee}$ states onto $\omega_{t}^{\vee}$ states, and finally, we give the correspondence result.

Given a $\mathrm{CHR}_{\vee}^{\text {brp }} \operatorname{program} P$, we create a $\mathrm{CHR}^{\vee} \operatorname{program}$ nondet $(P)$ as follows. For every rule in $P$ of the form

$$
(b p, r p):: r @ H^{k} \backslash H^{r} \Longleftrightarrow \text { guard } \mid b p_{1}:: B_{1} \vee \ldots \vee b p_{m}:: B_{m}
$$

nondet $(P)$ contains a rule

$$
r @ H^{k} \backslash H^{r}, \mathrm{bp}(b p) \Longleftrightarrow \operatorname{guard} \mid\left(\mathrm{bp}\left(b p_{1}\right), B_{1}\right) \vee \ldots \vee\left(\mathrm{bp}\left(b p_{m}\right), B_{m}\right)
$$

and for every rule of the form

$$
(b p, r p):: r @ H^{k} \backslash H^{r} \Longleftrightarrow \text { guard } \mid \text { body }
$$

where body does not contain a disjunction, nondet $(P)$ contains a rule

$$
r @ \mathrm{bp}(b p), H^{k} \backslash H^{r} \Longleftrightarrow \operatorname{guard} \mid \text { body }
$$


It is easy to see that given one bp/ 1 constraint in the initial goal, no state can be derived in which the CHR constraint store contains more than one such constraint. We incorporate the branch priorities of $P$ as constraints into nondet $(P)$ because they may appear in guards or rule bodies. In the following, we assume that no constraint identifier is used for the bp/1 constraint and that it is ignored by the propagation history. This can be realized by a source-to-source transformation in which constraint identifiers and the propagation history are made explicit.

Now we define a mapping function map from $\omega_{p}^{\vee}$ states to $\omega_{t}^{\vee}$ states.

$$
\begin{aligned}
\operatorname{map}(\{b p:: \sigma\} \uplus \Sigma)= & \operatorname{map}(b p:: \sigma) \uplus \operatorname{map}(\Sigma) \\
\operatorname{map}\left(b p::\langle G, S, B, T\rangle_{n}\right)= & \begin{cases}\left\langle G^{\prime}, S, B, T\right\rangle_{n} \quad \text { if } G \text { consists of a disjunction } \\
\langle G,\{\mathrm{bp}(b p)\} \cup S, B, T\rangle_{n} \text { otherwise }\end{cases} \\
& \text { where } G^{\prime} \text { is found by replacing each disjunct } \\
& b p_{i}:: B_{i} \text { in } G \text { by a disjunct }\left(\operatorname{bp}\left(b p_{i}\right), B_{i}\right)
\end{aligned}
$$

Theorem 1 (Correspondence). Given a $\mathrm{CHR}_{\vee}^{\mathrm{brp}}$ program $P$ and the corresponding $\mathrm{CHR}^{\vee}$ program nondet $(P)$, then for each transition $\Sigma \stackrel{\omega_{p}^{\vee}}{\longmapsto_{P}} \Sigma^{\prime}$, there exists a derivation $\operatorname{map}(\Sigma) \stackrel{\omega_{t}^{\vee}}{\longmapsto}$ nondet $(P) \operatorname{map}\left(\Sigma^{\prime}\right)$ and if $\Sigma$ is a final $\omega_{p}^{\vee}$ state, then $\operatorname{map}(\Sigma)$ is a final $\omega_{t}^{\vee}$ state.

Proof. Let there be given a transition $\Sigma \stackrel{\omega_{p}^{\vee}}{\longmapsto} \Sigma_{P}^{\prime}$. Each $\omega_{p}^{\vee}$ transition operates on a single (highest priority, unmarked) alternative $b p:: \sigma$, and replaces this alternative with one or more new alternatives. Let $\Sigma=\{b p:: \sigma\} \uplus \Sigma_{R}$ and let $\Sigma^{\prime}=\Sigma_{N} \uplus \Sigma_{R}$. We also have that $\operatorname{map}(\Sigma)=\operatorname{map}(b p:: \sigma) \uplus \operatorname{map}\left(\Sigma_{R}\right)$. Now a transition from $\Sigma$ to $\Sigma^{\prime}$ can be one of the following:

1a. Solve $\sigma=\langle\{c\} \uplus G, S, B, T\rangle_{n}$ with $c$ a built-in constraint, and so $\operatorname{map}(b p$ : $\sigma)=\langle\{c\} \uplus G,\{\mathrm{bp}(b p)\} \cup S, B, T\rangle_{n}$. Therefore, in $\operatorname{map}(\Sigma)$, the $\omega_{t}^{\vee}$ Derive transition is possible since the $\omega_{t}$ Solve transition applies to map $(b p:: \sigma)$. This results in the $\omega_{t}^{\vee}$ state $\left\{\langle G,\{\mathrm{bp}(b p)\} \cup S, c \wedge B, T\rangle_{n}\right\} \uplus \operatorname{map}\left(\Sigma_{R}\right)$ which is exactly $\operatorname{map}\left(\left\{b p::\langle G, S, c \wedge B, T\rangle_{n}\right\} \uplus \Sigma_{R}\right)$.

1b. Introduce $\sigma=\langle\{c\} \uplus G, S, B, T\rangle_{n}$ with $c$ a CHR constraint, and so map $(b p::$ $\sigma)=\langle\{c\} \uplus G,\{\operatorname{bp}(b p)\} \cup S, B, T\rangle_{n}$. Therefore, in $\operatorname{map}(\Sigma)$, the $\omega_{t}^{\vee}$ Derive transition is possible since the $\omega_{t}$ Introduce transition applies to $\operatorname{map}(b p$ :: $\sigma)$. This results in the $\omega_{t}^{\vee}$ state $\left\{\langle G,\{c \# n, \mathrm{bp}(b p)\} \cup S, B, T\rangle_{n+1}\right\} \uplus \operatorname{map}\left(\Sigma_{R}\right)$ which is exactly $\operatorname{map}\left(\left\{b p::\langle G,\{c \# n\} \cup S, c \wedge B, T\rangle_{n+1}\right\} \uplus \Sigma_{R}\right)$.

1c. Apply $\sigma=\left\langle\emptyset, H_{1} \uplus H_{2} \uplus S, B, T\right\rangle_{n}$ and $\operatorname{so} \operatorname{map}(b p:: \sigma)=\langle\emptyset,\{\mathrm{bp}(b p)\} \uplus$ $\left.H_{1} \uplus H_{2} \uplus S, B, T\right\rangle_{n}$. $P$ contains a rule of the form

$$
\left(b p^{\prime}, r p\right):: r @ H_{1}^{\prime} \backslash H_{2}^{\prime} \Longleftrightarrow g \mid C
$$

and there exists a matching substitution $\theta$ such that $\operatorname{chr}\left(H_{1}\right)=\theta\left(H_{1}^{\prime}\right)$, $\operatorname{chr}\left(H_{2}\right)=\theta\left(H_{2}^{\prime}\right), b p=\theta\left(b p^{\prime}\right), \mathcal{D} \models B \rightarrow \bar{\exists}_{B}(\theta \wedge g), \theta(r p)$ is a ground 
arithmetic expression and $t=\left\langle\mathrm{id}\left(H_{1}\right), \mathrm{id}\left(H_{2}\right), r\right\rangle \notin T$. Assume $C$ consists of a disjunction, then nondet $(P)$ contains a rule of the form

$$
r @ H_{1}^{\prime} \backslash H_{2}^{\prime}, \mathrm{bp}\left(b p^{\prime}\right) \Longleftrightarrow g \mid C^{\prime}
$$

where $C^{\prime}$ is found by replacing all $\mathrm{CHR}_{\vee}^{\mathrm{brp}}$ disjuncts of the form $b p_{i}:: B_{i}$ by $\mathrm{CHR}^{\vee}$ disjuncts of the form $\left(\mathrm{bp}\left(b p_{i}\right), B_{i}\right)$. Given this rule, the $\omega_{t}$ Apply transition applies to state $\operatorname{map}(b p:: \sigma)$. In particular, we can use $\theta$ as the matching substitution. Therefore, the $\omega_{t}^{\vee}$ Derive transition applies to state $\operatorname{map}(\Sigma)$, resulting in the state $\left\{\left\langle C^{\prime}, H_{1} \cup S, \theta \wedge B, T \cup\{t\}\right\rangle_{n}\right\} \uplus \operatorname{map}\left(\Sigma_{R}\right)$ where $t=\left\langle\operatorname{id}\left(H_{1}\right), \operatorname{id}\left(H_{2}\right), r\right\rangle$. This corresponds to the state $\operatorname{map}\left(\Sigma^{\prime}\right)=\operatorname{map}(\{b p::$ $\left.\left.\left\langle C, H_{1} \cup S, \theta \wedge B, T \cup\{t\}\right\rangle_{n}\right\} \uplus \Sigma_{R}\right)$. The case that $C$ does not contain a disjunction is similar. The difference is that the $\mathrm{bp} / 1$ constraint is not removed and the rule body remains unchanged.

2. Split $\sigma=\left\langle\left\{b p_{1}:: G_{1} \vee \ldots \vee b p_{m}:: G_{m}\right\}, S, B, T\right\rangle_{n}$ and $\operatorname{so} \operatorname{map}(b p:: \sigma)=$ $\left\langle\left\{\left(\mathrm{bp}\left(b p_{1}\right), G_{1}\right) \vee \ldots \vee\left(\mathrm{bp}\left(b p_{m}\right), G_{m}\right)\right\}, S, B, T\right\rangle_{n}$. In $\operatorname{map}(\Sigma)$, the $\omega_{t}^{\vee}$ Split transition applies, resulting in the state $\left\{\left\langle\left\{\mathrm{bp}\left(b p_{1}\right)\right\} \uplus G_{1}, S, B, T\right\rangle_{n}, \ldots\right.$, $\left.\left\langle\left\{\mathrm{bp}\left(b p_{m}\right)\right\} \uplus G_{m}, S, B, T\right\rangle_{n}\right\} \uplus \operatorname{map}\left(\Sigma_{R}\right)$. In each of the $m$ first alternatives, the Introduce transition applies, introducing the bp/1 constraint into the CHR constraint store. After these introductions, the resulting state equals $\left\{\left\langle G_{1},\left\{\mathrm{bp}\left(b p_{1}\right)\right\} \cup S, B, T\right\rangle_{n}, \ldots,\left\langle G_{m},\left\{\mathrm{bp}\left(b p_{m}\right)\right\} \cup S, B, T\right\rangle_{n}\right\} \uplus \operatorname{map}\left(\Sigma_{R}\right)$. This state equals map $\left(\Sigma^{\prime}\right)$ since $\Sigma^{\prime}=\left\{b p_{1}::\left\langle G_{1}, S, B, T\right\rangle_{n}, \ldots, b p_{m}::\left\langle G_{m}, S, B\right.\right.$, $\left.\left.T\rangle_{n}\right\} \uplus \Sigma_{R}\right)$.

3. Drop $\sigma=\langle G, S, B, T\rangle_{n}$ and $\mathcal{D} \models \neg \bar{\exists}_{\emptyset} B$. Since the map function does not change the built-in constraint store, the $\omega_{t}^{\vee}$ Drop transition applies to $\operatorname{map}(\Sigma)$ resulting in the state $\operatorname{map}\left(\Sigma_{R}\right)$. The result of applying the Drop transition to $\Sigma$ is the state $\Sigma_{R}$ and so the resulting states correspond.

4. Mark This transition does not change the result of the map function and so $\operatorname{map}(\Sigma)=\operatorname{map}\left(\Sigma^{\prime}\right)$.

This proves the first part of the theorem. For the second part, consider a final $\omega_{p}^{\vee}$ state $\Sigma$. Such a state consists of a set of alternatives, each of which is marked. An alternative is marked only if no other transition applies. This means (amongst others) that the goal of such an alternative is empty and its built-in constraint store is consistent. Once an alternative is marked, it remains unchanged. Now consider the $\omega_{t}^{\vee}$ state $\operatorname{map}(\Sigma)$. If an $\omega_{t}^{\vee}$ transition applies to this state, then this must be a Derive transition because the map function only adds a bp/1 constraint to the CHR constraint store of each alternative with an empty goal and so the Split and Drop transitions are not applicable. Let $\sigma$ be the alternative in $\operatorname{map}(\Sigma)$ that is replaced by a Derive transition. Since the goal of $\sigma$ is empty, the $\omega_{t}$ transition corresponding to the Derive transition must be an Apply transition, firing a rule of the form

$$
r @ H_{1}^{\prime} \backslash H_{2}^{\prime}, \mathrm{bp}\left(b p^{\prime}\right) \Longleftrightarrow g \mid C^{\prime}
$$

Let $\theta(r)$ be the fired rule instance, then the CHR constraint store of state $\sigma$ must contain sets of constraints $H_{1}$ and $H_{2}$ matching the heads $H_{1}^{\prime}$ and $H_{2}^{\prime}$, as well as 
a constraint $\mathrm{bp}(b p)$ that matches with $\mathrm{bp}\left(b p^{\prime}\right)$. Moreover, the built-in constraint store of $\sigma$ entails the guard $g$ in conjunction with the matching substitution $\theta$. Now, let $b p:: \sigma^{\prime}$ be the $\omega_{p}^{\vee}$ alternative in $\Sigma$ that maps on $\omega_{t}$ state $\sigma$. By definition, the CHR constraint store of $\sigma^{\prime}$ contains the constraints in $H_{1}$ and $H_{2}$, and its built-in constraint store and propagation history are equal to the ones of $\sigma$. The $\mathrm{CHR}_{\vee}^{\text {brp }}$ program $P$ contains a rule

$$
\left(b p^{\prime}, r p\right):: r @ H_{1}^{\prime} \backslash H_{2}^{\prime} \Longleftrightarrow g \mid C
$$

for which holds that rule instance $\theta(r)$ can fire given the branch priority, CHR and built-in constraint stores and propagation history of $\omega_{p}^{\vee}$ alternative $b p:: \sigma^{\prime}$. Potentially, $\theta(r)$ is not the highest priority applicable rule instance in $b p:: \sigma^{\prime}$, but then another rule instance can fire, and so this also implies that $\Sigma$ is not a final $\omega_{p}^{\vee}$ state. So we conclude that a non-final $\omega_{t}^{\vee}$ state corresponds to a nonfinal $\omega_{p}^{\vee}$ state, which proves the second part of the theorem.

\section{Specifying Common Search Strategies}

In this section, we show how different search strategies can be implemented in $\mathrm{CHR}_{\vee}^{\mathrm{brp}}$. In Section 4.1, we look at uninformed strategies such as depthfirst, breadth-first and depth-first iterative deepening. It is shown that a $\mathrm{CHR}_{\vee}^{\mathrm{rp}}$ program (i.e., one without branch priorities) can be automatically translated into a $\mathrm{CHR}_{\vee}^{\text {brp }}$ program that implements these search strategies. Next, in Section 4.2, we consider informed search strategies such as best-first search, $\mathrm{A}^{*}$ and limited discrepancy search. Finally, in Section 4.3, we show how different strategies can be combined, with as an example a mixture of depth- and breadth-first search.

\subsection{Uninformed Search Strategies}

Depth-first and breadth-first search In order to implement depth-first or breadth-first search, we transform each $\mathrm{CHR}_{\vee}^{\mathrm{rp}}$ rule of the form

$$
r p:: H^{k} \backslash H^{r} \Longleftrightarrow \text { guard } \mid B_{1} \vee \ldots \vee B_{n}
$$

into a $\mathrm{CHR}_{\vee}^{\text {brp }}$ rule

$$
(D, r p):: H^{k} \backslash H^{r} \Longleftrightarrow \text { guard } \mid(D+1):: B_{1} \vee \ldots \vee(D+1):: B_{n}
$$

The branch priorities correspond to the depth in the search tree: $\mathcal{B P}=\mathbb{N}$ and $b p_{0}=0$. We define the branch priority order $\preceq$ as follows:

- for depth-first search, $D_{1} \preceq D_{2} \Leftrightarrow D_{1} \leq D_{2}$

- for breadth-first search, $D_{1} \preceq D_{2} \Leftrightarrow D_{1} \geq D_{2}$

Now, the branch priorities are such that for depth-first search, the deeper alternative has a higher priority, whereas for breadth-first search, the more shallow alternative has a higher priority. 
Example 2 (4-queens (ctd.)). The 4-queens solver given in Example 1 can be extended with branch and rule priorities as follows.

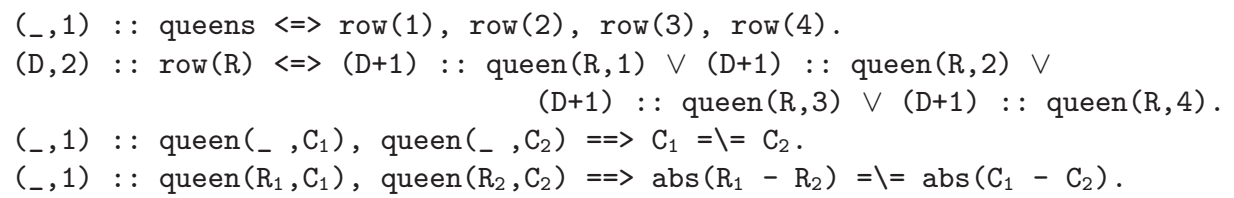

The branch priorities implement depth-first or breadth-first search, depending on the $\preceq$ order used. The rule priorities ensure that (further) labeling is done only after consistency checking. The derivation starts with the following initial $\mathrm{CHR}_{\vee}^{\text {brp }}$ state: $\left\{0::\langle\{\text { queens }\}, \emptyset, \text { true }, \emptyset\rangle_{1}\right\}$.

The implementation of depth- and breadth-first search given above is still nondeterministic with respect to alternatives at equal depth. We can implement a deterministic left-to-right version of depth-first or breadth-first search as follows. Take as branch priorities sequences of integers $\left(\mathcal{B P}=\mathbb{N}^{*}\right.$ and $\left.b p_{0}=\epsilon\right)$. The length of the sequence denotes the depth in the search tree, and the $i^{\text {th }}$ element in the sequence denotes the number of the branch taken at level $i$.

The order over these priorities is defined as

$$
(D, r p):: H^{k} \backslash H^{r} \Longleftrightarrow \text { guard } \mid(D+[1]):: B_{1} \vee \ldots \vee(D++[n]):: B_{n}
$$

with

$$
\begin{aligned}
L_{1} \succeq L_{2} & \Leftrightarrow \\
& \left(\text { length }\left(L_{1}\right)>\text { length }\left(L_{2}\right)\right) \vee\left(\text { length }\left(L_{1}\right)=\operatorname{length}\left(L_{2}\right) \wedge L_{1} \leq^{d} L_{2}\right)
\end{aligned}
$$

for depth-first search and

$$
\begin{aligned}
L_{1} \succeq L_{2} & \Leftrightarrow \\
& \left(\text { length }\left(L_{1}\right)<\text { length }\left(L_{2}\right)\right) \vee\left(\text { length }\left(L_{1}\right)=\operatorname{length}\left(L_{2}\right) \wedge L_{1} \leq^{d} L_{2}\right)
\end{aligned}
$$

for breadth-first search. Here $\leq^{d}$ is the lexicographic or dictionary order.

Depth-limited search and depth-first iterative deepening Depth-limited search is a variant of depth-first search in which search tree nodes are only expanded up to a given depth bound. It is an incomplete search in that it is not able to find solutions beyond this depth bound. Amongst others, depth-limited search is used in iterative deepening search. It can be implemented in $\mathrm{CHR}_{\vee}^{\text {brp }}$ by using the depth-first search program given in the previous paragraph, extended with the following rule:

$(D, 1):: \operatorname{limit}(D) \Leftrightarrow$ false. 
This rule ensures that any alternative at the depth limit fails. Its rule priority ensures the rule is tried before any other rule. ${ }^{6}$ Here, the depth limit is given by an appropriate limit/1 constraint which is to be added to the initial goal.

Depth-first iterative deepening [21] consists of iteratively running depthlimited search, increasing the depth limit in each run. Iterative deepening can be implemented by adding the following rule:

$\left(\_, 1\right):: \operatorname{deepen}(D) \Leftrightarrow 1:: \operatorname{limit}(\mathrm{D}) \vee 0:: \operatorname{deepen}(\mathrm{D}+1)$.

Instead of a limit/1 constraint, the goal is extended with a deepen(1) constraint. Using the above approach may lead to an infinite loop in which the depth limit keeps increasing in case the search tree is finite but contains no solutions. The reason is that it is not possible to distinguish between failure because the depth limit is reached, and failure because the entire search tree has been traversed and no solutions were found. In Section 5.4 we return to this issue and show how conflict-directed backjumping can solve this problem. More precisely, it is shown that if failure is independent of the depth limit, there is no need to change it.

Iterative broadening Iterative broadening [15] works similar to iterative deepening, but instead of using a depth limit that is iteratively increased, the number of branches starting at any given node is limited, and this limit increases over the iterations. The domain of branch priorities $\mathcal{B P}=\{\langle D, B\rangle \mid D, B \in \mathbb{N}\}$. A node with branch priority $\langle D, B\rangle$ is at depth $D$ in the search tree, and is the $B^{t h}$ alternative of its parent node. We use as initial branch priority $b p_{0}=\langle 0,1\rangle$ and we define the $\preceq$ relation as follows:

$$
\left\langle D_{1},-\right\rangle \preceq\left\langle D_{2},-\right\rangle \Leftrightarrow D_{1} \leq D_{2}
$$

Now, the code below implements iterative broadening.

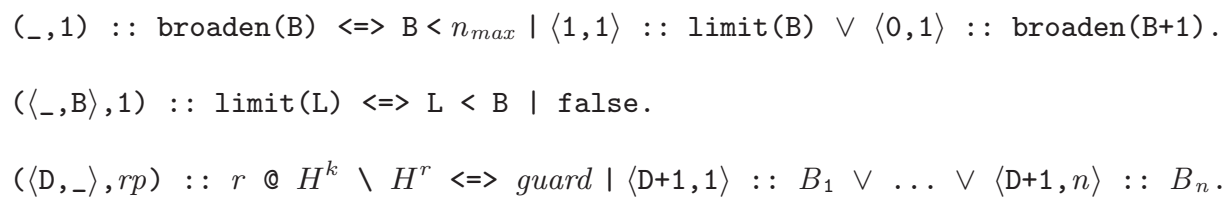

In the above, $n_{\max }$ is an upperbound on the number of alternatives in a rule body, and is used to ensure termination. The depth-first strategy ensures that the sub-tree for a given breadth limit is completely traversed before increasing this breadth limit (by means of a broaden/1 constraint). The second component of the branch priorities of the alternatives created by the first rule, is of no importance, as long as it is less than the breadth limit. We extend the initial goal with a broaden(1) constraint to start the process.

\subsection{Informed Search Strategies}

Informed strategies take problem dependent heuristics into account.

\footnotetext{
${ }^{6}$ This may require increasing the rule priorities of all other rules by one.
} 
Limited and depth-bounded discrepancy search The limited discrepancy search (LDS) [19] strategy is similar to best-first search. It is designed for boolean constraint satisfaction problems in which the values of each variable are ordered according to some heuristic. The idea behind LDS is that if following the heuristic does not work, then it is probable that a solution can be found by violating the heuristic only a limited number of times. Each violation of the heuristic is called a discrepancy, and the algorithm consists of first trying those alternatives with at most one discrepancy, then the ones with at most two discrepancies and so on until a solution is found. Let there be given the following $\mathrm{CHR}_{\vee}^{\mathrm{rp}}$ labeling rule:

$r p:: \operatorname{domain}\left(\mathrm{X},\left[\mathrm{V}_{1}, \mathrm{~V}_{2}\right]\right) \Leftrightarrow\left(\mathrm{X}=\mathrm{V}_{1}\right) \vee\left(\mathrm{X}=\mathrm{V}_{2}\right)$.

where the values in the domain are ordered according to the heuristic, i.e., $\mathrm{V}_{1}$ is preferred over $\mathrm{V}_{2}$. In $\mathrm{CHR}_{\vee}^{\mathrm{brp}}$, we can write this labeling rule as follows:

$(\mathrm{D}, r p):: \operatorname{domain}\left(\mathrm{X},\left[\mathrm{V}_{1}, \mathrm{~V}_{2}\right]\right) \Leftrightarrow \mathrm{D}::\left(\mathrm{X}=\mathrm{V}_{1}\right) \vee(\mathrm{D}+1)::\left(\mathrm{X}=\mathrm{V}_{2}\right)$.

The branch priority represents the number of discrepancies: $\mathcal{B P}=\mathbb{N}$. Initially, there is no discrepancy: $b p_{0}=0$. In each choice point, the discrepancy remains unchanged if we follow the heuristic, and increased by one if we deviate from this heuristic. The alternatives with fewer discrepancies are explored first: $P_{1} \preceq$ $P_{2} \Leftrightarrow P_{1} \geq P_{2}$.

Note that the description of LDS in [19] uses depth-first search combined with a limit on the number of discrepancies. Here, we use a form of best-first search where alternatives with less discrepancies are preferred. A variant of LDS in which the number of discrepancies is actually bounded, can be expressed in the same way as how we express depth-limited search, i.e., by introducing a limit/1 constraint and adding a rule

$(D, 1):: \operatorname{limit}(D-1) \Leftrightarrow>$ false.

Another variant of LDS, called depth-bounded discrepancy search (DDS) [35] combines LDS with iterative deepening. It is best characterized as a depth-first search that only allows discrepancies below a certain depth, that is iteratively increased. As for depth-first search, the branch priority denotes the depth in the search tree $(\mathcal{B P}=\mathbb{N} \cup\{-1\})$ and we start at the root $\left(b p_{0}=0\right)$. The order over these priorities is the same as for depth-first search.

The original program is transformed to:

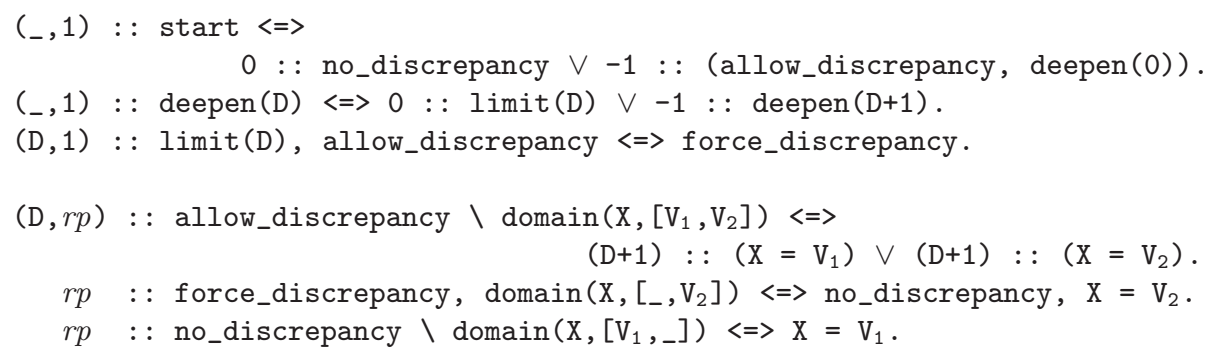


Here, the limit/1 constraint represents the current depth limit. Above this limit any number of discrepancies are allowed (allow_discrepancy/0), while below the limit no discrepancies are allowed (no_discrepancy/0). It gets complicated when we are at the current depth limit. Let us first focus on the iterative deepening part. The deepen/1 constraint drives the iterative loop of installing successively increasing depth limits. The extra element $-1 \in \mathcal{B P}$ is the minimal priority, which ensures that we only install the next depth limit after the current one has been fully explored. Each successive iteration should only produce additional solutions, which have not been found in preceding iterations. Hence, all solutions should exploit the increased depth-limit and have a discrepancy at that depth. The force_discrepancy/0 constraint makes sure this happens. By adding the start/0 constraint to the goal, we get the process going.

Similar to the case of depth-first iterative deepening, the depth limit keeps increasing if either the problem is overconstrained, or we require all solutions. Again, using conflict-directed backjumping remedies this problem. Only if failure happens in a state in which the constraint store contains a force_discrepancy $/ 0$ or no_discrepancy/0 constraint (justified by a limit/1 constraint), the depth limit is changed.

A* and iterative deepening $\mathbf{A}^{*}$ The $\mathrm{A}^{*}$ algorithm [18] consists of using bestfirst search to find an optimal solution in a constrained optimization problem. Let the branch priorities be such that $p:: \sigma$ is better than $p^{\prime}:: \sigma^{\prime}$ for successful final states $\sigma$ and $\sigma^{\prime}$, if and only if $p \succeq p^{\prime}$; and $\{p:: \sigma\} \stackrel{\omega_{p}^{\vee}}{\longmapsto}{ }_{P}^{*} \Sigma$ implies $p \succeq p_{i}$ for all $p_{i}:: \sigma_{i} \in \Sigma$. Under these conditions, the first answer found (using find_next) is also an optimal solution.

Example 3 (Shortest path distance). Consider the following program for computing the shortest path distance between two nodes in a directed graph.

$\left(\_, 1\right):: \operatorname{path}(\mathrm{V}, \mathrm{V}) \Leftrightarrow$ true

$(\mathrm{D}, 2)::$ neighbors $(\mathrm{V}, \mathrm{CU}) \backslash \operatorname{path}(\mathrm{V}, \mathrm{W}) \Leftrightarrow 1:$ : $\operatorname{branches}(\mathrm{W}, \mathrm{D}, \mathrm{CU})$.

$\left(\_, 1\right):: \operatorname{branches}(\mathrm{W}, \mathrm{D},[\mathrm{C}-\mathrm{U} \mid \mathrm{CUs}]) \Leftrightarrow$

$(\mathrm{D}+\mathrm{C}):: \operatorname{path}(\mathrm{U}, \mathrm{W}) \vee 1:: \operatorname{branches}(\mathrm{W}, \mathrm{D}, \mathrm{CUs})$.

$\left({ }_{-}, 1\right)::$ branches $\left(_{-},,_{-},[]\right) \Leftrightarrow$ false.

Here, the neighbors $/ 2$ constraints represent the edges of the graph: for each node $V$, there exists a neighbors $(V, C U s)$ constraint with $C U s$ a list containing a pair $C-U$ for each edge from node $V$ to node $U$ with cost $C$. The initial goal consists of a single path $/ 2$ constraint whose arguments are the nodes for which we wish to compute the shortest path distance. The branch priorities denote the distance between the initial start node and the node represented by the first argument of the path $/ 2$ constraint currently in the store, or, eventually in a successful final state, the distance between the initial start and end nodes. We have $\mathcal{B P}=\mathbb{N}, b p_{0}=0$ and $D_{1} \preceq D_{2} \Leftrightarrow D_{1} \geq D_{2}$. 
Iterative deepening $\mathrm{A}^{*}\left(\mathrm{ID}-\mathrm{A}^{*}\right)$ is a combination of $\mathrm{A}^{*}$ and depth-first iterative deepening. It consists of depth-first search in part of the search tree, only considering nodes whose cost function does not exceed a certain threshold value. If no solution is found, the threshold is increased by the minimal amount needed to include an unseen search tree node. We can easily implement a variant of ID-A* in which the threshold is increased by some fixed amount, similar to how we implement depth-first iterative deepening. However, increasing the threshold with the minimal amount needed to include the lowest cost unseen node, falls outside of the scope of our framework as it requires communication between different branches of the search tree.

\subsection{Combining Search Strategies}

Now we show that the $\mathrm{CHR}_{\vee}^{\text {brp }}$ language is expressive enough to formulate complex search strategies. In particular, with an appropriate choice of priorities and orderings, compositions of the previous search strategies can be expressed. In this subsection, we give two examples of such strategy compositions.

Example 4. Consider we want to use breadth-first search, but only up to a certain depth, e.g. so as not to exceed available memory. Beyond the depth limit, the search should switch to a depth-first strategy.

To implement this more complex strategy, we use the same branch priorities as the ones used for depth-first and breadth-first search in Section 4.1. The following definition for the $\preceq$ relation is used

$$
D_{1} \preceq D_{2} \Leftrightarrow\left(D_{2} \leq T \wedge D_{2} \leq D_{1}\right) \vee\left(T \leq D_{1} \leq D_{2}\right)
$$

where $T$ is the depth threshold which is given by the user. In words, beyond the threshold, the deeper alternative is preferred, whereas below the threshold, the more shallow alternative is preferred. An alternative whose depth is below the threshold is preferred over one whose depth is beyond the threshold.

In the second example, we show how to traverse the states resulting from some Split transitions in a depth-first order, and others in a breadth-first order.

Example 5. Let us assume a $\mathrm{CHR}_{\vee}^{\mathrm{rp}}$ program containing the following two binary labeling rules:

$1:$ : label_df @ df_domain $\left(X,\left[\mathrm{~V}_{1}, \mathrm{~V}_{2}\right]\right) \Leftrightarrow \mathrm{X}=\mathrm{V}_{1} \vee \mathrm{X}=\mathrm{V}_{2}$.
$1:$ : label_bf @ bf_domain $\left(\mathrm{X},\left[\mathrm{V}_{1}, \mathrm{~V}_{2}\right]\right) \Leftrightarrow \mathrm{X}=\mathrm{V}_{1} \vee \mathrm{X}=\mathrm{V}_{2}$.

Furthermore assume we want the labeling by rule label_df to proceed in depthfirst, and the labeling by rule label_bf in breadth-first order. We define a depthfirst subtree as part of the search tree in which all internal nodes correspond to depth-first splits. A breadth-first subtree is similarly defined. Comparing alternatives within a depth-first or breadth-first subtree is straightforward. For alternatives across such subtrees, we proceed as follows. 
Let there be two alternatives $A_{1}$ and $A_{2}$, let $N^{1}=\left[N_{1}^{1}, \ldots, N_{n_{1}}^{1}\right]$ be the sequence of nodes on the unique path from the root of the search tree, to (but excluding) alternative $A_{1}$, and let $N^{2}=\left[N_{1}^{2}, \ldots, N_{n_{2}}^{2}\right]$ be the sequence of nodes on the path from the root to alternative $A_{2}$. Let $i_{1} \in\left\{1, \ldots, n_{1}+1\right\}$ be the first index for which holds that node $N_{i_{1}}$ corresponds to a breadth-first (depthfirst) split or $n_{1}+1$ if no such index exists. In particular, the nodes in the subsequence $\left[N_{1}^{1}, \ldots, N_{i_{1}-1}^{1}\right]$ all correspond to depth-first (breadth-first) splits. Let $i_{2} \in\left\{1, \ldots, n_{2}+2\right\}$ be similarly defined. If $i_{1}>i_{2}\left(i_{1}<i_{2}\right)$ then alternative $A_{1}$ has priority over alternative $A_{2}$ and if $i_{1}<i_{2}\left(i_{1}>i_{2}\right)$ then the opposite holds. Finally, if $i_{1}=i_{2}$, we compare the depths of the first depth-first (breadthfirst) splits in sequences $\left[N_{i_{1}}^{1}, \ldots, N_{n_{1}}^{1}\right]$ and $\left[N_{i_{2}}^{2}, \ldots, N_{n_{2}}^{2}\right]$ and so on until either the depths are different or there are no more nodes to consider, in which case both alternatives have an equal priority.

Figure 1 shows an example search tree including its depth-first and breadthfirst subtrees. Node 2 (as well as all alternatives on the edge from Node 1 to Node 2) has priority over Node 1 as both are part of the same depth-first subtree and Node 2 is deeper than Node 1 . Node 1 has priority over Nodes 3 and 4 , even though the latter are deeper in the global search tree. The reasoning is as follows. The depth of the first breadth-first split in the path from the root node to respectively Nodes 1, 3 and 4 all equals 2 (for Node 1, there is no such split). In the remaining subpaths (for Node 1, this path is empty), the depth of the first depth-first split equals 1 for Node 1, 3 for Node 3 and 2 for Node 4. Therefore, Node 1 has priority over Nodes 3 and 4 . Furthermore, Node 4 has priority over Node 3. Finally, by transitivity, Node 2 also has priority over Nodes 3 and 4.

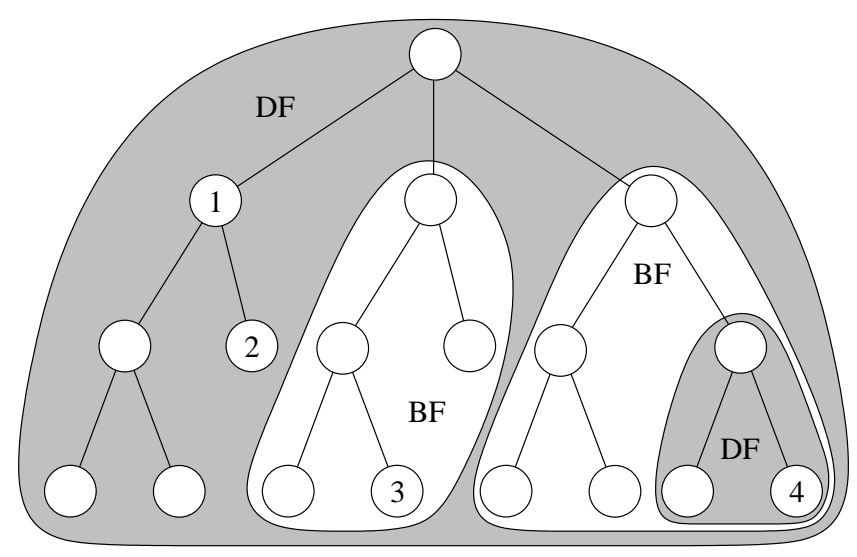

Fig. 1. Search tree for mixed depth-and-breadth-first search

In $\mathrm{CHR}_{\vee}^{\text {brp }}$, we can model the above described preference relation using the following rules. 


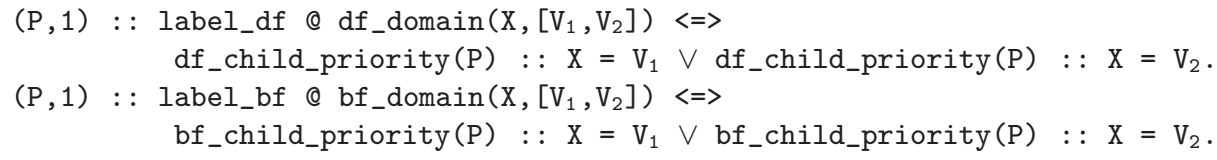

where the functions df_child_priority and bf_child_priority are implemented as follows: ${ }^{7}$

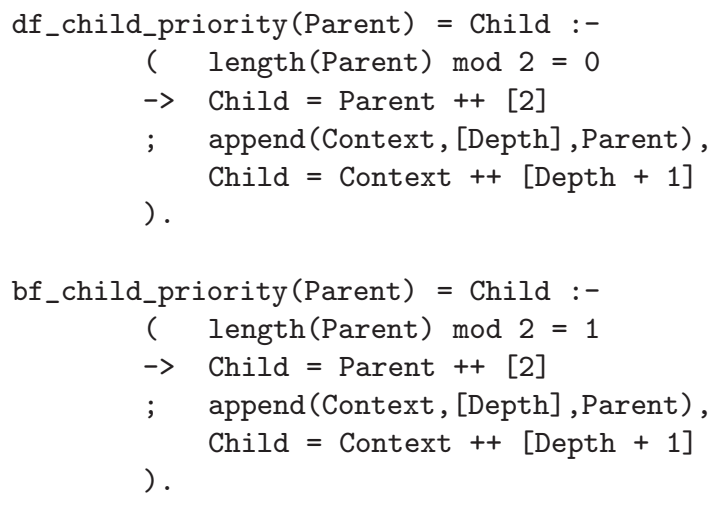

The initial branch priority is set to $\epsilon$. We define the order relation $\succeq$ as follows:

$$
L_{1} \succeq L_{2} \Leftrightarrow L_{1} \succeq^{d} L_{2}
$$

where

$$
\begin{aligned}
& {\left[H_{1} \mid T_{1}\right] \succeq^{d} \epsilon} \\
& {\left[H_{1} \mid T_{1}\right] \succeq^{d}\left[H_{2} \mid T_{2}\right] \Leftrightarrow H_{1}>H_{2} \vee\left(H_{1}=H_{2} \wedge T_{1} \succeq^{b} T_{2}\right)}
\end{aligned}
$$

and

$$
\begin{aligned}
\epsilon & \succeq^{b}\left[H_{2} \mid T_{2}\right] \\
{\left[H_{1} \mid T_{1}\right] } & \succeq^{b}\left[H_{2} \mid T_{2}\right] \Leftrightarrow H_{1}<H_{2} \vee\left(H_{1}=H_{2} \wedge T_{1} \succeq^{d} T_{2}\right)
\end{aligned}
$$

The branch priority of an alternative is a list of depths of the first depth-first or breadth-first split in consecutive subpaths from the root to the alternative as defined earlier. ${ }^{8}$ For example, in Figure 1, the branch priority equals [2] for Node 1, [3] for Node 2, [2,3] for Node 3, and [2,2,2] for Node 4. It is assumed that the root node is a depth-first node; if necessary, a dummy depth-first split can be created to ensure this is true. One can now verify that

$$
[3] \succeq[2] \succeq[2,2,2] \succeq[2,3]
$$

\footnotetext{
${ }^{7}$ We use Mercury syntax here [31], wich supports a functional notation for predicates; $p\left(\bar{X}, X_{n}\right):-$ body is equivalent to $p(\bar{X})=X_{n}:-$ body.

8 The list is implicitly assumed to end with an infinite sequence ones $=[1 \mid$ ones $]$.
} 


\section{Look Back Schemes: Conflict-Directed Backjumping}

In [38], Wolf et al. use justifications and conflict sets to define an extended and refined operational semantics $\omega_{r}^{\vee^{*}}$, which supports look back schemes like conflictdirected backjumping (CBJ) [24] and dynamic backtracking [14] as opposed to standard chronological backtracking. In this section, we show how their approach can be combined with our framework. More precisely, we propose an extended version of the $\omega_{p}^{\vee}$ semantics that supports conflict-directed backjumping, and discuss the correctness and optimality of this extension. We note that the benefits of CBJ are limited when strong constraint propagation and a good variable ordering heuristic is used (see for example [8]). We have chosen not to support dynamic backtracking, as it requires changing the shape of the search tree, which may conflict with the execution order imposed by the rule priorities. Moreover, for efficiency it requires an adaptive version of CHR [37].

\section{$5.1 \quad$ Justifications and Labels}

Justifications are introduced in the CHR context in [37] and used for the purpose of finding no-goods in conflict-directed backjumping in [38]. In that context, justifications consist of the choices that caused a given constraint to hold. The $\omega_{p}$ semantics can easily be extended to support justifications by annotating each constraint in the goal, CHR constraint store, and built-in constraint store with a justification. A constraint $c$ with justification $J$ is denoted by $c^{J}$ and we write just $\left(c^{J}\right)=J$. The transitions of the extended $\omega_{p}$ semantics are given as part of the extended priority semantics of $\mathrm{CHR}_{\vee}^{\mathrm{brp}}$ in the next subsection.

In case of depth-first search, conflicts can be uniquely described using only the search tree levels at which the conflicting choices were made. This is the approach taken in [38]. For more general search strategies, we need a more precise specification of the choices involved in a conflict. Therefore, we introduce a labeling scheme for search tree nodes that allows us to distinguish between such nodes, and to decide whether a given node is a descendant of another. In general, we can use as labels the branch priorities used in the (deterministic) left-to-right versions of depth- and breadth-first search proposed in Section 4.1.

\subsection{The Extended Priority Semantics of $\mathrm{CHR}_{\vee}^{\mathrm{brp}}$}

In analogy with [38], we extend the $\omega_{p}^{\vee}$ semantics into the $\omega_{p}^{\vee^{\star}}$ semantics, whose states are tuples $\langle K, S\rangle$ where $K$ is the conflict set, a set of no-goods, i.e., justifications of failures; and $S$ is a set of (marked and unmarked) $\omega_{p}$ states extended with a branch priority and branch label: $S=\left\{b p_{1}:: b l_{1} @ \sigma_{1}, \ldots, b p_{n}:: b l_{n} @ \sigma_{n}\right\}$. By slight abuse of notation, we use $b l$ to refer to just the label, or to the labeled alternative $b p:: b l @ \sigma$, depending on the context.

While in case of depth-first search, backjumping consists of skipping a series of alternatives, in general, it requires pruning of the search tree. This is because the alternatives that are skipped with depth-first search are exactly the (remaining) children of the deepest conflict, while in general, these children may be 
scheduled for later resolution when using a different search strategy and hence they cannot be skipped.

The transitions of the extended priority semantics of $\mathrm{CHR}_{\vee}^{\mathrm{brp}}$ are given in Table 4. The Backjump transition implements what corresponds to a multi-step backjump in [38]. It works by constructing a new failed alternative as a child of the node to which is jumped back. If this alternative is the only remaining child of its parent, then the next applicable transition will again be a Backjump transition. Otherwise, a Backtrack transition will follow, which just removes the failed alternative. The latter is treated as a special case in [38], by the single-step backjump transition.

Example 6 (6-queens). Consider the 6-queens problem where we are labeling the last row and all previously rows have their queens set as in the figure below.

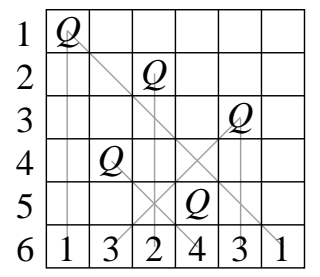

We see that all positions in the last row are conflicting with some previously set queen. For each position, we have given the first row which contains a conflicting queen. Note that some positions conflict with more than one queen: for example for the second column, these are the queens in rows 3 and 4 . Because the fifth row participates in none of the conflicts, we can jump back to the queen in the fourth row and move her, instead of considering alternative places for the queen in the fifth row.

In $\mathrm{CHR}_{\vee}^{\mathrm{brp}}$, we can use the following solver for the 6 -queens problem:

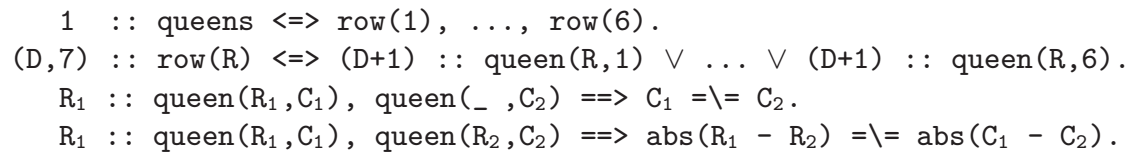

This solver differs from the one in Example 2 in that conflicts with queens in early rows are preferred above those with queens in later rows. This preference is imposed by using the row number of the conflicting queen as rule priority. ${ }^{9}$ It ensures that we jump back as far as possible.

In the remainder of this example, we use a simplified notation for $\omega_{p}^{\vee^{\star}}$ states. More precisely, we represent an alternative of the form $b p:: b l @\langle G, S, B, T\rangle_{n}$ as $b p:: \operatorname{chr}(S) \cup B$, i.e., we do not show the branch label (which is assumed to be equal to the branch priority), goal, propagation history or next free identifier, and we represent CHR constraints without their identifier. We assume a depthfirst strategy which implies that it is sufficient to use the depth in the search tree

\footnotetext{
${ }^{9}$ A conflict between the queens in rows $i$ and $j(i<j)$ can be detected by a rule instance of priority $i$, and a symmetric one of priority $j$. Only the first one fires.
} 


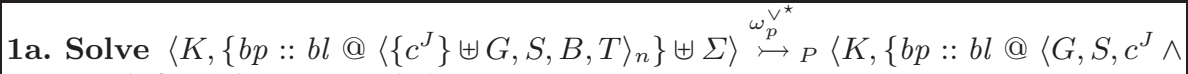
$\left.\left.B, T\rangle_{n}\right\} \uplus \Sigma\right\rangle$ if max_bp $(\Sigma) \preceq b p$ and $c$ is a built-in constraint.

1b. Introduce $\left\langle K,\left\{b p \quad:: \quad b l @\left\langle\left\{c^{J}\right\} \uplus G, S, B, T\right\rangle_{n}\right\} \uplus \Sigma\right\rangle{\stackrel{\omega_{p}^{\vee^{*}}}{\longmapsto}}_{P}\langle K,\{b p$ $\left.\left.b l @\left\langle G,\left\{c^{J} \# n\right\} \cup S, B, T\right\rangle_{n+1}\right\} \uplus \Sigma\right\rangle$ if $\max \_b p(\Sigma) \preceq b p$ and $c$ is a CHR constraint.

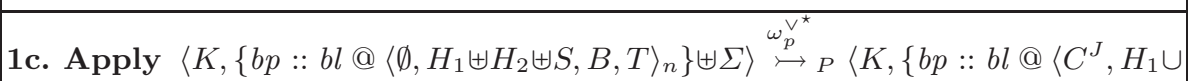
$\left.\left.S, \theta \wedge B, T \cup\{t\}\rangle_{n}\right\} \uplus \Sigma\right\rangle$ if max_bp $(\Sigma) \preceq b p$ and where $P$ contains a rule of priority $r p$ of the form

$$
\left(b p^{\prime}, r p\right):: r @ H_{1}^{\prime} \backslash H_{2}^{\prime} \Longleftrightarrow g \mid C
$$

and a matching substitution $\theta$ exists such that $\operatorname{chr}\left(H_{1}\right)=\theta\left(H_{1}^{\prime}\right), \operatorname{chr}\left(H_{2}\right)=$ $\theta\left(H_{2}^{\prime}\right), b p=\theta\left(b p^{\prime}\right), \mathcal{D} \models B \rightarrow \bar{\exists}_{B}(\theta \wedge g), \theta(r p)$ is a ground arithmetic expression and $t=\left\langle\operatorname{id}\left(H_{1}\right), \operatorname{id}\left(H_{2}\right), r\right\rangle \notin T$. Furthermore, no rule of priority $r p^{\prime}$ and substitution $\theta^{\prime}$ exists with $\theta^{\prime}\left(r p^{\prime}\right)<\theta(r p)$ for which the above conditions hold. The justification $J=\operatorname{just}\left(H_{1}\right) \cup$ just $\left(H_{2}\right) \cup$ just $(E)$ where $E$ is a minimal subset of $B$ for which holds that $\mathcal{D} \models E \rightarrow \bar{\exists}_{E}(\theta \wedge g)$.

2. Split $\langle K,\{b p:: b l @ \sigma\} \uplus \Sigma\rangle{\stackrel{\omega_{p}^{\vee^{*}}}{\longmapsto}}_{P}\left\langle K,\left\{b p_{1}:: b l_{1} @ \sigma_{1}, \ldots, b p_{m}:: b l_{m} @ \sigma_{m}\right\} \uplus \Sigma\right\rangle$ where $\sigma=\left\langle\left(b p_{1}:: G_{1} \vee \ldots \vee b p_{m}:: G_{m}\right)^{J} \wedge G, S, B, T\right\rangle_{n}, \max \_b p(\Sigma) \preceq b p$, and $\sigma_{i}=\left\langle G_{i}^{J \cup\left\{b l_{i}\right\}} \wedge G, S, B, T\right\rangle_{n}$ for $1 \leq i \leq m$.

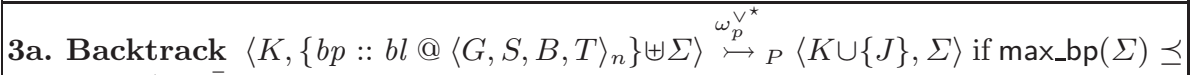
$b p, \mathcal{D} \models \neg \bar{\exists}_{\emptyset} B$, and there exists at least one alternative in $\Sigma$ that is a descendant of the parent of $b l$. Here, $J$ is the justification of the inconsistency of $B$.

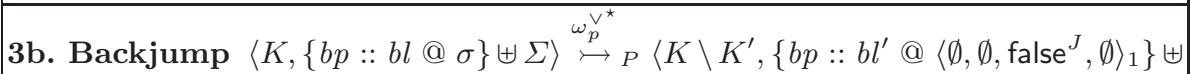
$\left.\Sigma^{\prime}\right\rangle$ if max_bp $(\Sigma) \preceq b p, \sigma$ is a failed $\omega_{p}$ state, and there exists no other alternative in $\Sigma$ that is a descendant of the parent of $b l$. Let $K^{\prime}$ be the justifications in $K$ that involve $b l$ or one of its siblings. A new no-good $J$ is created by merging these justifications, removing the labels of $b l$ and its siblings. Now let $b l^{\prime}$ be the deepest alternative in $J$. We find $\Sigma^{\prime}$ by removing from $\Sigma$ all descendants of $b l^{\prime}$.

4. Mark $\langle K,\{b p:: b l @ \sigma\} \uplus \Sigma\rangle \stackrel{\omega_{p}^{\vee^{*}} \longmapsto_{P}}{b^{*}}\left\langle K,\left\{b p:: b l @ \sigma^{\star}\right\} \uplus \Sigma\right\rangle$ if $\max \_b p(\Sigma) \preceq b p$ and no other $\omega_{p}^{\vee^{\star}}$ transition applies.

Table 4. Transitions of $\omega_{p}^{\vee^{\star}}$ 
as branch labels. Now using our simplified representation, the $\omega_{p}^{\vee^{\star}}$ state right before labeling the last row looks as follows:

$$
\left\langle K,\left\{\left(5:: Q_{5} \cup\left\{\operatorname{row}(6)^{\emptyset}\right\}\right)\right\} \cup \Sigma\right\rangle
$$

where $Q_{4}=\left\{\right.$ queen $(1,1)^{\{1\}}$, queen $(2,3)^{\{2\}}$, queen $(3,5)^{\{3\}}$, queen $\left.(4,2)^{\{4\}}\right\}, Q_{5}=$ $Q_{4} \cup\left\{\right.$ queen $\left.(5,4)^{\{5\}}\right\}$ and $\Sigma=\left\{\left(5:: Q_{4} \cup\left\{\right.\right.\right.$ queen $\left.\left.(5,5)^{\{5\}}, \operatorname{row}(6)^{\emptyset}\right\}\right),\left(5:: Q_{4} \cup\right.$ $\left\{\right.$ queen $\left.\left.\left.(5,6)^{\{5\}}, \operatorname{row}(6)^{\emptyset}\right\}\right)\right\} \cup \Sigma^{\prime}$. The contents of $K$ and $\Sigma^{\prime}$ is not relevant to our presentation. The labeling rule replaces this $\omega_{p}^{\vee^{\star}}$ state by

$$
\left\langle K,\left\{\left(6:: Q_{5} \cup\left\{\text { queen }(6,1)^{\{6\}}\right\}\right), \ldots,\left(6:: Q_{5} \cup\left\{\text { queen }(6,6)^{\{6\}}\right\}\right)\right\} \cup \Sigma\right\rangle
$$

Now each of the queens on row 6 conflicts with a queen in an earlier row. These conflicts lead to failures that are justified by the conflicting constraints' labels. After having dealt with columns 1 to 5 , the resulting $\omega_{p}^{\vee^{\star}}$ state is

$$
\left\langle K \cup\{\{1,6\},\{3,6\},\{2,6\},\{4,6\},\{3,6\}\},\left\{\left(6:: Q_{5} \cup\left\{\text { queen }(6,6)^{\{6\}}\right\}\right)\right\} \cup \Sigma\right\rangle
$$

So far, we have only used Backtrack transitions to deal with failures. The last alternative position on the sixth row again leads to failure, this time with justification $\{1,6\}$. Now, the Backjump transition applies, which forms a new justification by merging the ones involving the sixth row. This new justification equals $\{1,2,3,4\}$. The Backjump transition removes all (two) remaining alternatives on the fifth row and creates a new failed alternative, resulting in the state

$$
\left\langle K,\left\{\left(6:: \text { false }^{\{1,2,3,4\}}\right)\right\} \cup \Sigma^{\prime}\right\rangle
$$

after which (in this case) a Backtrack transition follows and the next alternative on row 4 is tried:

$$
\left\langle K \cup\{\{1,2,3,4\}\}, \Sigma^{\prime}\right\rangle
$$

\subsection{Correctness and Optimality Issues}

We now discuss three issues concerning the correctness and optimality of conflictdirected backjumping in $\mathrm{CHR}^{\vee}$ (and $\mathrm{CHR}_{\vee}^{(\mathrm{b}) \mathrm{rp}}$ ). Firstly, for correctness, we need to impose restrictions on the programs for which we can use conflict-directed backjumping. In particular, we require that a program is confluent with respect to the $\omega_{t}^{\vee}$ semantics. The following theorem states the correctness of the $\omega_{p}^{\vee}$ semantics.

Theorem 2. For a given program $P$ whose non-deterministic version $\operatorname{nondet}(P)$ is confluent with respect to the $\omega_{t}^{\vee}$ semantics, it holds that $\Sigma_{0} \stackrel{\omega_{p}^{\vee^{*}}}{\longmapsto}{ }_{P}^{*} \Sigma_{n}$ with $\Sigma_{n}$ a final $\omega_{p}^{\vee^{\star}}$ state, if and only if $\Sigma_{0} \stackrel{\omega_{p}^{\vee}}{\longmapsto}{ }_{P}^{*} \Sigma_{n}$. 
Proof (sketch). The main proof obligation consists of showing that the Backjump transition is correct, i.e., that it does not discard any solutions. We show that this is true given that the existence of a failing $\omega_{t}^{\vee}$ derivation for a state $\operatorname{map}(\Sigma)$ in the non-deterministic version nondet $(P)$ of a program $P$, implies that all such derivations fail.

Consider a node $N$, all of whose children have failed, where the failures are justified by justifications $J_{1}, \ldots, J_{n}$. A justification $J_{i}(1 \leq i \leq n)$ is a set of branch labels. After the last child of $N$ fails, a Backjump transition applies under $\omega_{p}^{\vee^{*}}$. This transition consists of merging the justifications $J_{1}, \ldots, J_{n}$ into a new justification $J$ by uniting the respective sets of branch labels, discarding the labels of children of $N$.

Let $b l$ be the branch label of the deepest alternative in $J$. The branch labeled $b l$ ends in a node, i.e., a state in which a Split transition applies (see Section 2.3), which is preceded by an Apply transition, firing some rule instance $\theta(r)$. Let $b p:: b l @ \sigma$ be the alternative right before $\theta(r)$ fired. In this alternative and under the $\omega_{t}^{\vee}$ semantics, we can also fire the rule instance that lead to the creation of node $N$, say $\theta^{\prime}\left(r^{\prime}\right)$, in state $\operatorname{map}(b p:: \sigma)$ using program nondet $(P)$ (see Section 3.3). The reasoning is that none of the children of $N$ depend on constraints derived after state $\sigma$ as their branch labels would otherwise have been part of the justification $J$.

Now there exists a failing derivation $D=\{\operatorname{map}(b p:: \sigma)\} \stackrel{\omega_{t}^{\vee}}{\rightleftarrows} *$ nondet $(P) \emptyset$ which consists of first firing rule instance $\theta^{\prime}\left(r^{\prime}\right)$ and then repeating the derivations that lead to the failure of each of the children of node $N$. If we have that the existence of a failing derivation such as $D$, implies that all derivations starting in the same state also fail, then we also have that all children of the ending node of branch $b l$ fail under the $\omega_{p}^{\vee^{*}}$ semantics, and so we can safely discard them as is done in the Backjump transition. We call the above property conflict preservation. It is a notion weaker than confluence in the sense that all solutions should only be the same in case one of them is a failure. In practice we can use the notion of confluence, for which a decidable test exists in case of a terminating program $[1],{ }^{10}$ to decide whether or not we can apply conflict-directed backjumping to a program. Figure 2 shows how the failing $\omega_{t}^{\vee}$ derivation $D$ can be constructed, starting in state $\operatorname{map}(b p:: \sigma)$, and justifying the backjump.

The following example shows how solutions can be missed in case a program is not confluent.

Example \%. Consider the $\mathrm{CHR}_{\vee}^{\mathrm{rp}}$ program below, with initial goal $\{\mathrm{g}\}$.

$1:$ : a, e $\Leftrightarrow$ false.

$1:: a, f \Leftrightarrow$ false.

$1:: a, d \Leftrightarrow$ true.

$2:: \mathrm{g}==>\mathrm{a} \vee \mathrm{b}$.

$\overline{10}$ This test is defined for the $\omega_{t}$ semantics, but can easily be extended towards the $\omega_{t}^{\vee}$ semantics. 


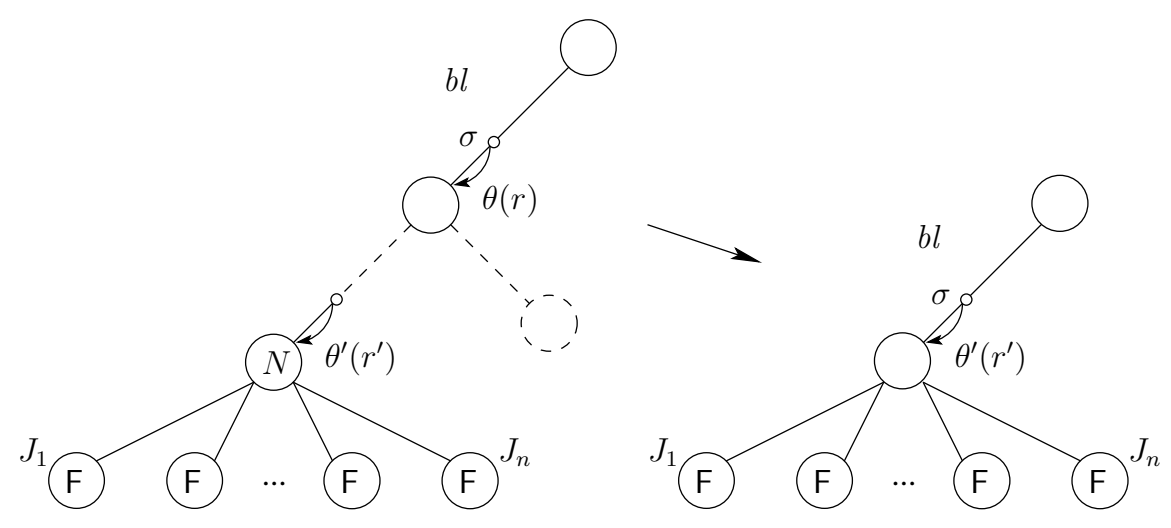

Fig. 2. Construction of a failing derivation, justifying a backjump.

$3:: \mathrm{g}==>\mathrm{c} \vee \mathrm{d}$.

$4:: g==>\vee f$.

We have left out branch priorities as they are not needed for the example. Assume a first labeling step chooses a, then $\mathrm{c}$ is chosen, and finally both e and $\mathrm{f}$ fail due to a and hence regardless of the second choice point (in which $c$ was chosen). However, we cannot jump back to the first choice, because if we choose $\mathrm{d}$ in the second choice point, the a constraint is 'consumed' and both e and $f$ are consistent. The problem is that the program is not confluent w.r.t. the $\omega_{t}^{\vee}$ semantics. For example, if the constraint store in a given state consists of the CHR constraints a, e and $d$, then the result can be either a failed state, or a state in which the constraint store contains the constraint e.

A second issue is concerned with optimality. In general, when it is detected that a constraint is in conflict with some other constraints, there might be other sets of conflicting constraints as well. In the conflict-directed backjumping algorithm, it is assumed that testing for conflicts follows the order in which the variables are instantiated. In general, we prefer conflicts with constraints that appear closer to the root of the search tree.

In the 6-queens program of Example 6, we dealt with this issue by giving conflicts with earlier queens a higher (rule) priority. This approach easily extends towards other finite domain constraint solvers that do not apply look-ahead schemes. However, in general, a conflict may involve multiple CHR and builtin constraints, each of which has its own justification. Therefore, it might not always be clear which rule instance is preferred. We could for example minimize the depth of the deepest choice, the number of choices, or the sum of the depths of the choices involved in a conflict. Some strategies maximize the depth of the first backjump, while others maximize for example the total number of nodes skipped. 
A final issue is that choice points should preferably be created only after all constraint propagation and consistency checking is completed. Otherwise, it is possible that a failure is independent of the last choice made. By assigning a low rule priority to labeling rules, we reach consistency before further labeling.

\subsection{Iterative Deepening Revisited}

In Section 4.1 the iterative deepening strategy is implemented. However, as noted, there is a problem with termination. In iterative deepening, the depth limit should only increase if the search tree contains an unexplored subtree. So, when a depth-limited search fails, we are interested in why it fails. This type of information is not available in the $\omega_{p}^{\vee}$ semantics, but it is available in the $\omega_{p}^{\vee^{*}}$ semantics by means of the conflict set.

When a failure occurs because the depth limit is reached, this failure is due to the following rule:

$(D, 1):: \operatorname{limit}(D-1) \Leftrightarrow$ false.

The justification for the failure hence contains the justification of the limit/1 constraint. If however no failure occurs because the depth limit is reached, then the justification for the failure does not contain the one of the limit/1 constraint and therefore, the second alternative of the rule that generated this constraint, namely

$\left(\_, 1\right):: \operatorname{deepen}(D) \Leftrightarrow 1:: \operatorname{limit}(D) \vee 0:: \operatorname{deepen}(D+1)$.

will be pruned by the Backjump transition.

Noteworthy is that the above approach only works if branch priorities are annotated with justifications themselves, and these justifications are taken into account when constraints rely on the branch priority. To simplify the presentation, we have ignored this in our description of the $\omega_{p}^{\vee^{*}}$ semantics in Section 5.2. We note that a constraint, asserted after firing some rule instance $\theta(r)$, should contain the justification of the branch priority only if either the firing of $\theta(r)$ depends on the branch priority, or the branch priority is used in the arguments of the constraint. If we do not take into account the justifications of the branch priority, then it may happen that a backjump is made to change the depth limit if all children of some node fail due to this depth limit, without taking into account that other nodes may be below the depth limit.

\section{Related Work}

Adding different search strategies to declarative languages, and in particular Constraint (Logic) Programming languages, has been done before. For a more thorough overview, see for example [13,28]. In [29] it is shown how search tree states can be encapsulated in the multi-paradigm language Oz. The encapsulation is implemented using a variable scoping mechanism and local versions of the constraint store, and allows the implementation of different search strategies. A 
generalization of this work is presented for the functional logic programming language Curry in [17]. An important aspect there is that the encapsulated search tree states are evaluated lazily.

Much related to our work is the OPL modeling language [32] in which search strategies consist of three parts: the exploration strategy, the limit strategy and the selection strategy. The exploration strategy consists of an evaluation function that assigns a score to search tree nodes, much like our branch priorities, combined with a procedure to decide when to switch to another node. The limit strategy imposes bounds on the time and space used for searching. Finally, the selection strategy determines which solutions are returned and can be used for instance to implement constrained optimization.

Constraint Logic Programming systems such as $\mathrm{ECL}^{i} \mathrm{PS}^{e}$ [34] or SICStus Prolog's clp (fd) [7] are usually limited by Prolog's built-in depth-first search mechanism. ${ }^{11}$ However, using language features such as the findall/3 predicate or blackboard primitives, other strategies can be implemented. For example, in Ciao Prolog [20], breadth-first and depth-first iterative deepening search are supported using a source transformation. In [6], the blackboard primitives of SICStus Prolog are used to implement intelligent backtracking in Prolog.

In all of the above mentioned work, the search strategy is stated independently of the program logic and therefore treats all search in a uniform way. Our approach supports both uniform and rule specific strategies, thereby allowing the use of different search strategies for different parts of the program.

Related work in the CHR context In the context of $\mathrm{CHR}^{\vee}$, Menezes et al. propose in [22] a $\mathrm{CHR}^{\vee}$ implementation for Java in which the search tree is made explicit and manipulated at runtime to improve efficiency. In particular, when a rule firing is independent of the choice made in a previous choice point, the result of this rule firing is valid for all alternatives in that choice point, and so by reordering the nodes in the search tree, some redundant work is avoided. In a sense, this reasoning takes into account justifications of constraints, i.e., those constraints that caused the derivation of any given constraint.

Justifications are introduced in CHR in the context of adaptive CHR [37], which extends CHR by supporting the incremental adaption of CHR derivations in the context of both constraint insertions and deletions. In [36], justifications, in particular for the built-in constraint false, are used to implement intelligent search algorithms such as conflict-directed backjumping and dynamic backtracking. A new operational semantics for $\mathrm{CHR}^{\vee}$, called the extended and refined operational semantics $\omega_{r}^{\vee^{\star}}$, which formally combines the concept of justifications with $\mathrm{CHR}^{\vee}$, is given in [38]. The semantics in fact implements conflict-directed backjumping. We extend the work in [38] by also supporting search strategies different from left-to-right depth-first in combination with conflict-directed backjumping, and by discussing optimality and correctness issues.

\footnotetext{
${ }^{11}$ Search strategies in these systems mostly consist of different ways of shaping the
} search tree, with the exploration strategy being fixed to left-to-right depth-first. 
In [25], it is proposed to transform the disjuncts in $\mathrm{CHR}^{\vee}$ into special purpose constraints which can be dealt with by an external search component. An example of such an external search component is the Java Abstract Search Engine (JASE), which is part of the Java Constraint Kit (JACK) [4], a CHR implementation for Java. Since Java does not offer native search in contrast with Prolog, Java-based CHR implementations need to implement their own search support. In practice, this often means more flexibility compared to the built-in search in Prolog. In particular, Prolog only supports a limited way of movement between nodes of the search tree, whereas in JASE, one can jump from one search tree node to another by means of trailing, copying or recomputation, as well as combinations of these methods.

\section{Conclusion}

To conclude, we summarize our contributions. In Section 3 we combined and extended two language extensions of $\mathrm{CHR}$, namely $\mathrm{CHR}^{\mathrm{rp}}$ and $\mathrm{CHR}^{\vee}$, into a flexible framework for execution control in CHR. In this framework, called $\mathrm{CHR}_{\vee}^{\mathrm{brp}}$, the propagation strategy is determined by means of rule priorities, whereas the search strategy is determined by means of branch priorities. In Section 4 , we have shown how various tree search strategies can be expressed in our framework. These strategies include uninformed search strategies such as depthfirst, breadth-first and depth-first iterative deepening (Section 4.1), informed strategies such as limited discrepancy search (Section 4.2), as well as combinations of different strategies (Section 4.3). Finally, in Section 5, we have adapted the work of [38] which proposes a combination of conflict-directed backjumping (CBJ) with $\mathrm{CHR}^{\vee}$, to our framework, by adding support for search strategies different from left-to-right depth-first. Moreover, we have established correctness and optimality conditions for this combination of CBJ with $\mathrm{CHR}_{\vee}^{\mathrm{brp}}$.

Future work A first topic for future work is the (efficient) implementation of our search framework in CHR systems. In particular, it is worth considering adding such search support to systems that currently do not offer search facilities like the K.U.Leuven JCHR [33] and CCHR [39] systems. Some issues that an optimized implementation should deal with are the use of specialized priority queues for e.g. depth-first and breadth-first search, and the choice between copying, trailing and recomputation (as well as combinations of these) for the task of jumping between search tree nodes (see also [27]).

It would also be interesting to have a high-level way to combine different search strategies. The approach we have taken in Section 4.3 is rather ad hoc, and in general it remains unclear what the priority domain, order relation, and priority assignments should look like.

Finally, we have already shown how information from successes (e.g., branch $\&$ bound in Section 3.2) and failures (e.g., conflict-directed backjumping in Section 5) in other branches of the search tree can be used to speed up the constraint solving process. It would be interesting to see how similar approaches 
can be used, for example to implement iterative deepening $\mathrm{A}^{*}$ or the dynamic variable ordering technique proposed in [23]. The latter consists of changing the variable to be labeled next after a backjump, taking into account the reason for the backjump.

\section{References}

1. Slim Abdennadher. Operational semantics and confluence of constraint propagation rules. In Gert Smolka, editor, 3rd International Conference on Principles and Practice of Constraint Programming, volume 1330 of Lecture Notes in Computer Science, pages 252-266. Springer, 1997.

2. Slim Abdennadher. A language for experimenting with declarative paradigms. In 2nd Workshop on Rule-Based Constraint Reasoning and Programming, 2000.

3. Slim Abdennadher. Rule Based Constraint Programming: Theory and Practice. Habilitation, Institut für Informatik, Ludwig-Maximilians-Universität München, 2001.

4. Slim Abdennadher, Ekkerhard Krämer, Matthias Saft, and Matthias Schmauss. JACK: A Java constraint kit. Electronic Notes in Theoretical Computer Science, 64, 2002.

5. Slim Abdennadher and Heribert Schütz. $\mathrm{CHR}^{\vee}$ : A flexible query language. In Troels Andreasen, Henning Christiansen, and Henrik Legind Larsen, editors, 3rd International Conference on Flexible Query Answering Systems, volume 1495 of Lecture Notes in Computer Science, pages 1-14. Springer, 1998.

6. Maurice Bruynooghe. Enhancing a search algorithm to perform intelligent backtracking. Theory and Practice of Logic Programming, 4(3):371-380, 2004.

7. Mats Carlsson, Greger Ottosson, and Björn Carlson. An open-ended finite domain constraint solver. In Hugh Glaser, Pieter H. Hartel, and Herbert Kuchen, editors, 9th International Symposium on Programming Languages: Implementations, Logics, and Programs, volume 1292 of Lecture Notes in Computer Science, pages 191-206. Springer, 1997.

8. Xinguang Chen and Peter van Beek. Conflict directed backjumping revisited. Journal of Artificial Intelligence Research, 14:53-81, 2001.

9. Leslie De Koninck, Tom Schrijvers, and Bart Demoen. Search strategies in CHR(Prolog). In Tom Schrijvers and Thom Frühwirth, editors, 3rd Workshop on Constraint Handling Rules, Report CW 452, pages 109-123. Dept. of Computer Science, K.U.Leuven, 2006.

10. Leslie De Koninck, Tom Schrijvers, and Bart Demoen. User-definable rule priorities for CHR. In Michael Leuschel and Andreas Podelski, editors, 9th International ACM SIGPLAN Conference on Principles and Practice of Declarative Programming, pages 25-36. ACM Press, 2007.

11. Gregory J. Duck, Peter J. Stuckey, María García de la Banda, and Christian Holzbaur. The refined operational semantics of Constraint Handling Rules. In Bart Demoen and Vladimir Lifschitz, editors, 20th International Conference on Logic Programming, volume 3132 of Lecture Notes in Computer Science, pages 90-104. Springer, 2004.

12. Thom Frühwirth. Theory and practice of Constraint Handling Rules. Journal of Logic Programming, 37(1-3):95-138, 1998.

13. Thom Frühwirth, Laurent Michel, and Christian Schulte. Constraints in procedural and concurrent languages. In Francesca Rossi, Peter van Beek, and Toby 
Walsh, editors, Handbook of Constraint Programming, Foundations of Artificial Intelligence, chapter 13, pages 453-494. Elsevier Science Publishers, 2006.

14. Matthew L. Ginsberg. Dynamic backtracking. Journal of Artificial Intelligence Research, 1:25-46, 1993.

15. Matthew L. Ginsberg and William D. Harvey. Iterative broadening. Artificial Intelligence, 55(2):367-383, 1992.

16. Michael Hanus. Adding Constraint Handling Rules to Curry. In Michael Fink, Hans Tompits, and Stefan Woltran, editors, 20th Workshop on Logic Programming, INFSYS Research Report 1843-06-02, pages 81-90. TU Wien, 2006.

17. Michael Hanus and Frank Steiner. Controlling search in declarative programs. In Catuscia Palamidessi, Hugh Glaser, and Karl Meinke, editors, 10th International Symposium on Programming Language Implementation and Logic Programming / 7th International Conference on Algebraic and Logic Programming, volume 1490 of Lecture Notes in Computer Science, pages 374-390. Springer, 1998.

18. Peter E. Hart, Nils J. Nilsson, and Bertram Raphael. A formal basis for the heuristic determination of minimum cost paths. IEEE Transactions on Systems Science and Cybernetics, 4(2):100-107, 1968.

19. William D. Harvey and Matthew L. Ginsberg. Limited discrepancy search. In 14th International Joint Conference on Artificial Intelligence, volume 1, pages 607-615. Morgan Kaufmann, 1995.

20. Manuel V. Hermenegildo, Francisco Bueno, Daniel Cabeza, Manuel Carro, Maria J. García de la Banda, Pedro López-García, and Germán Puebla. The CIAO multidialect compiler and system: An experimentation workbench for future (C)LP systems. In Paqui Lucio, Maurizio Martelli, and Marisa Navarro, editors, 1996 Joint Conference on Declarative Programming, APPIA-GULP-PRODE, pages 105-110, 1996.

21. Richard E. Korf. Depth-first iterative-deepening: An optimal admissible tree search. Artificial Intelligence, 27(1):97-109, 1985.

22. Luis Menezes, Jairson Vitorino, and Marcos Aurelio. A high performance $\mathrm{CHR}^{\vee}$ execution engine. In Tom Schrijvers and Thom Frühwirth, editors, 2nd Workshop on Constraint Handling Rules, Report CW 421, pages 35-45. Dept. of Computer Science, K.U.Leuven, 2005.

23. Henry Müller. Static and dynamic variable sorting strategies for backtrackingbased search algorithms. In Armin Wolf, Thom W. Frühwirth, and Marc Meister, editors, 19th Workshop on (Constraint) Logic Programming, volume 2005-01 of Ulmer Informatik-Berichte, pages 99-110, 2005.

24. Patrick Prosser. Hybrid algorithms for the constraint satisfaction problem. Computational Intelligence, 9:268-299, 1993.

25. Jacques Robin, Jairson Vitorino, and Armin Wolf. Constraint programming architectures: Review and a new proposal. Journal of Universal Computer Science, 13(6):701-720, 2007.

26. Tom Schrijvers and Bart Demoen. The K.U.Leuven CHR system: implementation and application. In Thom Frühwirth and Marc Meister, editors, First Workshop on Constraint Handling Rules: Selected Contributions, volume 2004-01 of Ulmer Informatik-Berichte, pages 1-5. Universität Ulm, 2004.

27. Christian Schulte. Comparing trailing and copying for constraint programming. In Danny De Schreye, editor, 1999 International Conference on Logic Programming, pages 275-289. MIT Press, 1999.

28. Christian Schulte and Mats Carlsson. Finite domain constraint programming systems. In Francesca Rossi, Peter van Beek, and Toby Walsh, editors, Handbook of 
Constraint Programming, Foundations of Artificial Intelligence, chapter 14, pages 495-526. Elsevier Science Publishers, 2006.

29. Christian Schulte, Gert Smolka, and Jörg Würtz. Encapsulated search and constraint programming in Oz. In Alan Borning, editor, 2nd International Workshop on Principles and Practice of Constraint Programming, volume 874 of Lecture Notes in Computer Science, pages 134-150. Springer, 1994.

30. Barbara M. Smith and Paula Sturdy. Value ordering for finding all solutions. In Leslie Pack Kaelbling and Alessandro Saffiotti, editors, 19th International Joint Conference on Artificial Intelligence, pages 311-316. Professional Book Center, 2005.

31. Zoltan Somogyi, Fergus Henderson, and Thomas Conway. The execution algorithm of Mercury, an efficient purely declarative logic programming language. Journal of Logic Programming, 29(1-3):17-64, 1996.

32. Pascal van Hentenryck, Laurent Perron, and Jean-François Puget. Search and strategies in OPL. Transactions on Computational Logic, 1(2):285-320, 2000.

33. Peter Van Weert, Tom Schrijvers, and Bart Demoen. K.U.Leuven JCHR: A userfriendly, flexible and efficient CHR system for Java. In Tom Schrijvers and Thom Frühwirth, editors, 2nd Workshop on Constraint Handling Rules, Report CW 421, pages 47-62. Dept. of Computer Science, K.U.Leuven, 2005.

34. Mark Wallace, Stefano Novello, and Joachim Schimpf. ECL ${ }^{i} \mathrm{PS}^{e}$ : A platform for constraint logic programming. ICL Systems Journal, 12(1):159-200, 1997.

35. Toby Walsh. Depth-bounded discrepancy search. In 15th International Joint Conference on Artificial Intelligence, volume 2, pages 1388-1395. Morgan Kaufmann, 1997.

36. Armin Wolf. Intelligent search strategies based on adaptive Constraint Handling Rules. Theory and Practice of Logic Programming, 5(4-5):567-594, 2005.

37. Armin Wolf, Thomas Gruenhagen, and Ulrich Geske. On the incremental adaptation of CHR derivations. Applied Artificial Intelligence, 14(4):389-416, 2000.

38. Armin Wolf, Jacques Robin, and Jairson Vitorino. Adaptive CHR meets CHR ${ }^{\vee}$ : An extended refined operational semantics for $\mathrm{CHR}^{\vee}$ based on justifications. Lecture Notes in Artificial Intelligence: Special Issue on Recent Advances in Constraint Handling Rules, ????:??-??, 2008.

39. Pieter Wuille, Tom Schrijvers, and Bart Demoen. CCHR: The fastest CHR implementation, in C. In Khalil Djelloul, Gregory J. Duck, and Martin Sulzmann, editors, 4th Workshop on Constraint Handling Rules, pages 123-137. U.Porto, 2007. 